\title{
手性高价碘试剂的发展及展望
}

\author{
蔡倩* 马浩文 \\ (暨南大学药学院 广州 510530)
}

\begin{abstract}
摘要 在过去几十年中, 高价碘化学已成为有机化学研究的重要领域. 高价碘化合物在多种类型的化学转化中, 展现 与过渡金属相似的反应性质. 而其温和的反应条件、低耗费、环境友好、低毒性等特点, 使高价碘化学引起了广泛的 研究兴趣, 并取得了巨大的进展. 手性的高价碘试剂或前体也得以发展并应用于一系列化学计量或催化的不对称反应. 近年来, 手性高价碘领域研究进展显著, 但也存在诸多不足. 在本综述中, 根据其结构特点以及发展的时间线, 对多种 类型的手性高价碘试剂和前体做一个总结, 这将有助于帮助本领域研究者更好地理解手性高价碘化学的发展以及不足 之处.
\end{abstract}

关键词 手性; 手性高价碘; 手性芳基碘; 不对称反应; 不对称催化

\section{Recent Advances of Chiral Hypervalent lodine Reagents}

\author{
Cai, Qian* Ma, Haowen \\ (College of Pharmacy, Jinan University, Guangzhou 510530)
}

\begin{abstract}
Hypervalent iodine chemistry has arose as an important field in organic chemistry in the past decades. Hypervalent iodine compounds, with reactivities similarly to transition metals in many different types of transformations, have attracted broad interests in organic community due to their practical advantages in the mild conditions, low costs, environmental benign and low toxicity. Great progresses have been made in this field. Chiral hypervalent iodine reagents or precursors have also been developed and utilized in a variety of asymmetric reactions in a stoichiometric or catalytic way. Important advances have been witnessed in the field of chiral hypervalent iodine chemistry in recent years. However, great limitations still exist. In this review, we have made a summary of different types of chiral hypervalent iodine reagents and precursors according to the characteristics of these compounds and the timeline. It may be helpful for the researchers to better understand the development and limitations of chiral hypervalent iodine chemistry.
\end{abstract}

Keywords chirality; chiral hypervalent iodine; chiral aryl iodide; asymmetric reaction; catalytic asymmetric synthesis

\section{1 引言}

近年来, 高价碘化学成为有机化学中的重要领域. 由于其反应条件温和、安全高效、环境友好、低耗费以 及操作简单等优点, 高价碘试剂引起了有机化学家的广 泛关注, 被应用于各类反应转化. 特别是近年来, 使用 催化量的芳基碘化物和化学计量的氧化剂现场生成高 价碘, 促进反应进行, 可以避免预先制备高价碘试剂的 不便, 从而使高价碘化学的研究更为深入, 在各类反应 中应用更为广泛 ${ }^{[1,2]}$.

随着高价碘化学研究的不断深入, 手性高价碘促进 的不对称转化, 也成为不对称合成研究中的一个重要领 域, 取得了许多重要进展 ${ }^{[3]}$. 但相对高价碘化学的蓬勃 发展而言, 手性高价碘促进的不对称反应研究进展仍然 相对缓慢. 特别是与过渡金属催化的不对称反应相比 较, 手性高价碘促进的不对称反应类型相对受限, 对映 选择性相对不高. 这一情况在很大程度上是由于手性高
价碘试剂或前体发展缓慢所致. 而对于反应机理认识不 足, 也对手性高价碘试剂或前体的改造优化造成了困 扰.

关于高价碘化学以及在不对称反应中的应用, 文献 中已有很多的报道, 相关的综述以及书籍进行了大量的 总结. 2011 年 Liang 和 Ciufolini ${ }^{[4]}$ 在综述中, 简单总结了 关于手性高价碘试剂的发展以及四类主要的不对称反 应(图 1, A D). 近几年来, 文献中关于手性高价碘的报 道迅速增多, 目前已报道的手性高价碘试剂或前体有数 十种, 但反应类型并未广泛拓展. 我们在 Liang 和 Ciufolini 的基础上稍加整理, 在本综述中, 主要将穿插 介绍取得重要进展的六类反应(图 1): (A)硫醚到亚砜的 不对称氧化; (B)羰基化合物的 $\alpha$ 位不对称官能化; (C)酚 类化合物的不对称去芳构化; (D)烯烃的不对称双官能团 化; (E)烯烃的不对称氧化官能化重排反应; (F)不对称氧 化偶联反应. 此外, 其它的一些不对称转化也有所涉及.

* E-mail: caiqian@jnu.edu.cn; Tel: 020-85225123

Received November 21, 2018; published January 28, 2019

Project supported by the National Natural Science Foundation of China (Nos. 21772066, 21572229) and Guangdong Special Support Program (No. 2017TX04R059).

项目受国家自然科学基金(Nos. 21772066, 21572229)以及广东省“特支”计划(No. 2017TX04R059)资助. 
目前文献中关于手性高价碘促进的各类不对称反 应已经有较多的综述总结. 在本文中, 我们不会对这些 不对称反应本身做详细的探讨和分析, 而主要将针对手 性高价碘试剂或前体的发展历史, 根据其骨架结构特点 及时间线, 做一个简单的总结.

(A)

$$
R^{-S} R^{\prime}
$$<smiles>CC(C)(C)O</smiles><smiles>[R][SH+]([R])[O-]</smiles>

(B)<smiles>[R]CC([R])=O</smiles>

(C)<smiles>[R]c1ccccc1OCCO[R20](=O)[O-]</smiles>

(D)<smiles>[R]C=C[R]</smiles>

(E)

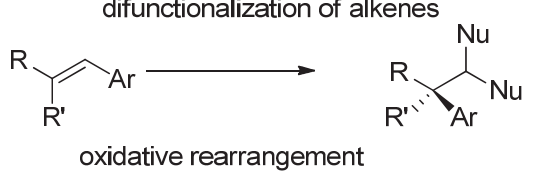

(F)

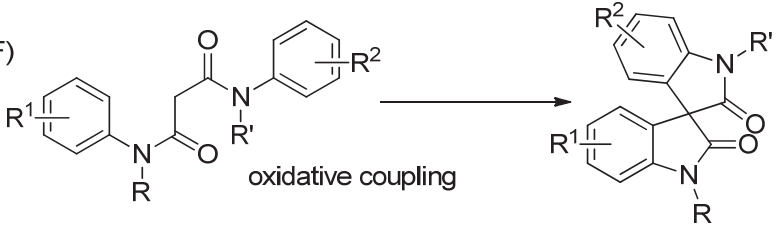

图 1 手性高价碘促进的几类主要不对称转化

Figure 1 Asymmetric transformations by hypervalent iodine reagents

\section{2 手性高价碘试剂的发展}

碘原子具有多种氧化态, 多种类型的手性碘, 包括 一价、三价、五价、七价碘等, 在手性高价碘化学中都 有所发展和应用. 在目前的报道中, 手性碘 $(\mathrm{V})$ 有少量 报道, 而手性碘(I/III)使用较为广泛. 特别是 Ochiai 和 Kita 等 ${ }^{[5]}$ 通过使用外加氧化剂氧化芳基碘化物现场生成 碘(III)的方法, 使原本需要当量使用的手性碘(III)试剂, 可以改为催化量的手性碘(I), 极大地方便了不对称反应 的研究.

手性高价碘主要可以分为两类: 一类是早期发展的 手性高价碘试剂, 手性部分类似于过渡金属催化中的手 性配体, 与碘原子相连(图 2, 式 I); 另一类则是整个分

$$
\mathrm{R}^{*}-\mathrm{O}^{\prime} \mathrm{I}-\mathrm{Ar}
$$$$
\text { I }
$$

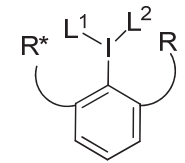

II

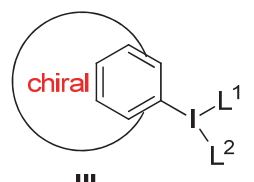

III
图 2 不同类型的手性高价碘试剂结构特点

Figure 2 Different types of chiral hypervalent iodine reagents and their general structures
子骨架具有手性. 后一种根据手性骨架的特点，又可以 分为具有柔性骨架和刚性骨架两类(图 2, 式 II 和 III). 下面我们将根据其结构特点, 具体的介绍这些手性高价 碘化合物.

\section{1 基于手性配体形式的高价碘化物}

最早的手性高价碘试剂可以追溯到 1907 年, Prib$\mathrm{ram}^{[6]}$ 制备了第一个手性高价碘试剂，二苯基碘鎓酒石 酸盐. 但这一手性高价碘试剂并没有在不对称合成中得 到关注和应用. 直到 1986 年, Imamoto 等 ${ }^{[7]}$ 报道了氧化 碘苯和 $L$-酒石酸酐现场生成七元环的手性三价碘烷(图 $3,1 \mathrm{a} \sim 1 \mathrm{c})$, 并应用到硫醚化合物转化为亚砜的不对称 氧化，才开启了手性高价碘促进的不对称反应研究大 门.在 Imamoto 等的这一工作中, 能够以中等至良好的 收率生成亚砜，对映选择性达到 53\% ee(如图 4).
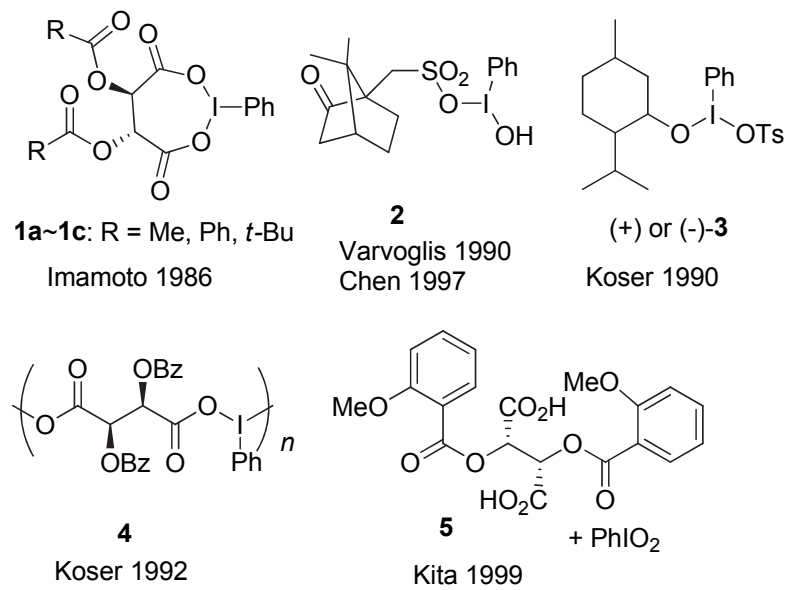

图 3 早期的手性高价碘试剂

Figure 3 Some early examples of chiral hypervalent iodine reagents<smiles>[R]C(=O)O[C@@H]1C(=O)OC(=O)[C@@H]1OC([R])=O</smiles><smiles>CCCCC(C)C</smiles><smiles>[R]C(=O)O[C@H]1C(=O)O[C@H](c2ccccc2)OC(=O)[C@H]1OC([R])=[18O]</smiles>
1a 1c: $\mathrm{R}=\mathrm{Me}, \mathrm{Ph}, t$-Bu

$$
\begin{aligned}
& \mathrm{R}^{1-S_{-}} \mathrm{R}^{2} \\
& \text { (R,R)-1a 1c } \\
& R^{1}=A y l, a k y l
\end{aligned}
$$$$
\mathrm{R}^{1}=\text { Aryl, alkyl }
$$$$
\mathrm{R}^{2}=\mathrm{Me}, t-\mathrm{Bu}
$$<smiles>[R][SH](C)=O</smiles>

$71 \% \sim 95 \%$ yield $5 \% \sim 53 \%$ ee
图 4 手性高价碘促进的硫梄不对称氧化为亚砜

Figure 4 Asymmetric oxidation of sulfides to sulfoxides by hypervalent iodine reagents

在 Imamoto 发展的这类芳基碘(III)试剂中, 酒石酸 部分类似于手性配体与碘苯结合，控制不对称反应进 行. 反应结束后，酒石酸部分与碘苯脱离. 整个反应过 程类似于过渡金属反应时配体与金属结合、脱除的过程.

之后其他研究小组如 Varvoglis ${ }^{[8]}$, Chen $^{[9]}$, Koser $^{[10,11]}$ 
等先后报道了几类类似的手性芳基碘(III)试剂(图 3, 2 4), 都是基于同样的手性配体形式的高价碘化物. 这些 手性芳基碘试剂在硫醚不对称氧化成亚砜的反应中, 都 具有很好的反应活性, 但反应的对映选择性或非对映选 择性不尽人意. 与 Imamoto 的催化剂相比, 并无提高.

在早期发展的这些手性高价碘化合物中, 手性元素 主要来源于 “手性池” 中的手性分子, 包括酒石酸、樟 脑磺酸、薄荷醇等. 由于手性取代基直接与高价碘原子 相连, 这类高价碘试剂在反应结束后, 含碘部分被还原, 将从整个手性骨架上脱离下来.

Kita 等 ${ }^{[12]}$ 选择酒石酸与五价碘试剂 $\mathrm{PhIO}_{2}$ 作用, 现 场生成手性的 $\mathrm{I}^{\mathrm{V}}$ 试剂. 在硫醚的不对称氧化反应中, 可 以良好到优秀的收率得到亚砜产物, 对映选择性最高可 以达到 $72 \% \mathrm{ee}$ (图 5). 这一反应中, 所采用的 $\mathrm{PhIO}_{2}$ 为 0.5 equiv., 手性源酒石酸衍生物为 $10 \mathrm{~mol} \%$. 这是第一 个催化的手性高价碘促进的不对称转化, 具有重要的意 义. 而且在这一反应中, 作者对可能的中间活化物种进 行了探讨, 认为芳环上的邻位甲氧基对形成的手性高价 碘物种可能存在配位作用, 从而有利于反应对映选择性 的提高. 这一推断, 对后来进一步的手性高价碘试剂或 前体的设计，显然具有重要的启发作用.

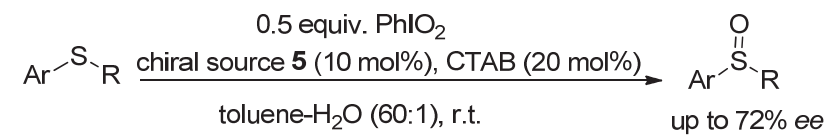

图 5 硫醚到手性亚砜的催化不对称氧化

Figure 5 Catalytic asymmetric oxidation of sulfides to sulfoxides by hypervalent iodine reagents

\section{2 基于中心手性柔性骨架的高价碘试剂或前体}

早期的手性高价碘试剂, 手性部分像配体一样与高 价碘相连, 随着反应进行, 高价碘结构被还原, 从而从 手性配体部分脱离. 而在 20 世纪 90 年代以后, 对手性 高价碘试剂或前体的认识不断深入, 基于手性骨架的高 价碘试剂或前体得以发展. 在本节中, 我们将首先介绍 基于中心手性柔性骨架的高价碘试剂及前体.

2.2.1 基于手性醇或醚的手性芳基碘(Koser 类手性碘 试剂)

$\operatorname{Koser}^{[13]}, W i r t h{ }^{[14]}$ 等在 1996 和 1997 年先后报道了
一类手性高价碘试剂, 代表性结构为图 6 中的 $6 \sim 8$. 在 这类试剂中，手性的醇或醚取代基连接在碘原子的邻位 芳基上. 这一改变具有显著的优点：一方面，手性中心 靠近碘原子的位置，使手性控制更为容易；另一方面， 在反应完成后，芳基碘片段仍然连接在手性骨架上，这 就为进一步重新氧化为高价碘试剂提供了可能. 也为后 来使用手性芳基碘化物作为催化剂, 外加氧化剂来现场 生成手性高价碘物种, 促进不对称转化奠定了基础.

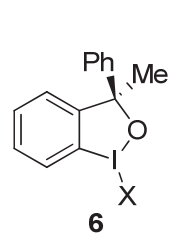<smiles>CCC(OC)c1cccc(C(CC)OC)c1B(O)O[Na]</smiles>

Koser 1996

Wirth 1997, 2001<smiles>CO[C@H](c1ccccn1)c1ccccc1I(OC(C)=O)OC(C)=O</smiles><smiles>CO[C@H](c1ccccn1)c1ccccc1I</smiles><smiles>[SnH3]OC(c1cn(Cc2ccccc2)nn1)c1ccccc1I</smiles>

Wirth 2014

Pericas \& Nachtseim 2017

图 6 Koser 类手性高价碘(I/III)试剂

Figure 6 Koser-type chiral hypervalent iodine reagents

Wirth 发展了一系列这类手性高价碘(I/III)试剂，代 表性例子包括手性高价碘(III)试剂 7 和 8 . 其中化合物 7 是一类具有 $\mathrm{C} 2$-对称的手性单芳基碘(III)试剂. Wirth 等 利用他们发展的手性芳基碘(III)试剂, 在羰基的 $\alpha$ 位不 对称官能团化、双键的不对称双官能团化反应中, 做了 大量的工作. 但大多情况下，对映选择性不高. 例如, 在酮的 $\alpha$-位磺酸酯化反应中，对映选择性只能达到 $30 \%$ $e e$ 左右，而在烯烃的双磺酸酯化反应中，对映选择性最 高也只能达到 $50 \%$ ee 左右. 一直到 2014 年, 他们 ${ }^{[15]}$ 发 展了另一个类似的手性碘(III)试剂 9, 在烯基化合物的 分子内双胺化环化反应中，才获得高的对映选择性，产 物最高获得 94\% ee. 当使用前体芳基碘(I)化合物 10 作 为催化剂, $\mathrm{NaBO}_{3}$ 作为氧化剂, 他们首次实现了催化的 烯烃分子内不对称双胺化过程，可以中等的收率得到产 物，对映选择性最高达到 86\%ee(图 7).

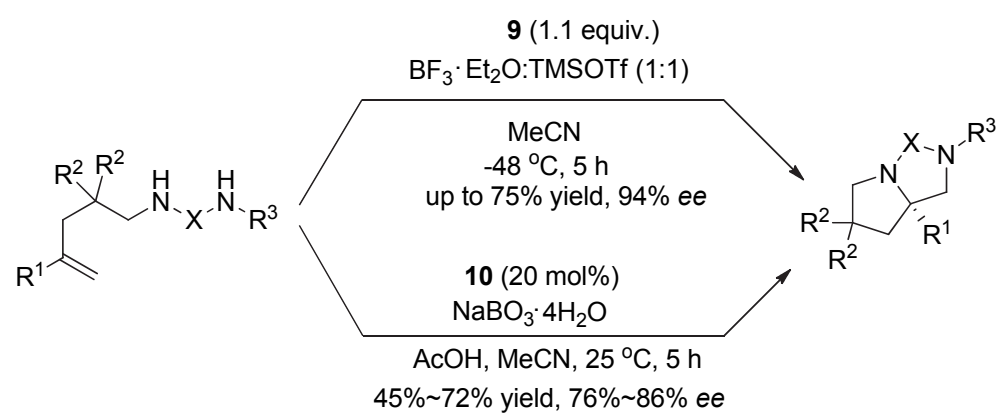

图 7 烯烃的不对称分子内双胺化环化反应

Figure 7 Enantioselective intramolecular diamination of alkenes 
2017 年, Pericàs 和 Nachtsheim 等 ${ }^{[16]}$ 以这类结构为基 础, 构建了一系列基于三氮唑的 Koser 类手性芳基碘化 合物，代表性结构为化合物 11. 他们采用这类芳基碘化 物, 以 $m-\mathrm{CPBA}$ 作为氧化剂, 实现了萘酚类化合物的氧 化 Kita 螺环内酯化反应. 当使用当量的芳基碘化物时, 可以中等到良好的收率得到产物, 对映选择性最高达到 $86 \%$ ee, 通过简单重结晶, 可以获得 $99 \%$ ee. 使用催化 量(15 mol\%)芳基碘化物时，产物的收率和对映选择性 有所降低(图 8). 这一结果虽然不如下面将介绍的一些 $\mathrm{C} 2$-对称手性芳基碘( $\mathrm{I} / \mathrm{III})$ 促进的 Kita 螺环内酯化反应, 但在目前报道的 C1-对称的手性芳基碘化物促进的同类 反应中, 对映选择性最高.

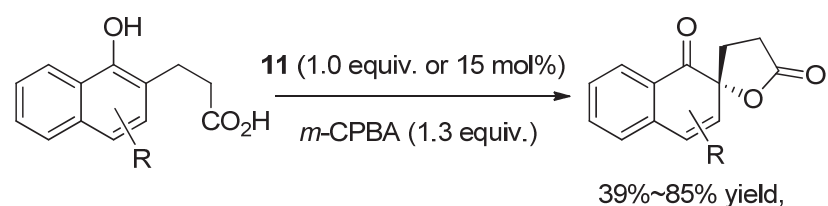
$58 \% \sim 86 \%$ ee

图 8 基于三氮唑的手性芳基碘促进的不对称 Kita 螺环内酯化反应 Figure 8 Enantioselective Kita spirocyclizations by triazole-based chiral iodoarenes

\subsection{2 基于乳酸的手性芳基碘(Fujita 和 Ishihara 类手性} 碘试剂)

早期所发展的手性芳基碘试剂或催化剂, 在不对称 转化中, 取得了重要的进展. 但与过渡金属催化的各类 反应相比, 对映选择性多差强人意. 但从 2007 年以后, Fujita 和 Ishihara 等基于乳酸, 发展了新类型的手性芳基 碘试剂或前体, 其结构通式如图 9 中的 IV 和 $\mathrm{V}$ 结构. 这 类手性碘试剂和前体, 在不对称转化中取得了巨大的成 功. 本节中我们进行具体介绍.<smiles></smiles><smiles>[X]C(=O)C([R])Oc1cc([R])cc(OC([R])C([R])=O)c1I</smiles>

图 9 基于乳酸的手性芳基碘结构通式

Figure 9 General structures of chiral aryl iodine reagents (I/III) developed from lactic acid
2007 年, Fujita 等 ${ }^{[17]}$ 最先通过邻碘苯酚与乳酸的 Mitsunobu 反应，在芳基碘的邻位引入乳酸手性片段， 发展了基于乳酸的新的手性碘(III)试剂 $12 \mathrm{a} \sim 12 \mathrm{~b}$. 他们 将这类手性碘(III)试剂用于高烯丙醇酯的双键分子内不 对称双官能团化环化反应，取得了中等程度的对映选择 性，最高达到 $64 \%$ ee. 但大多情况下，由于副反应存在， 反应的收率较低(图 10).

尽管 Fujita 以乳酸为手性源发展的芳基碘(III)试剂 在不对称反应中的初次表现似乎并不比以往的手性芳 基碘(I/III)更为出色, 但引起了 Ishihara 等的关注. Ishihara 对 Fujita 发展的手性高价碘化合物做了进一步的改 进，最终迎来了手性高价碘化学领域的重大突破.

2010 年, Ishihara 等 ${ }^{[18]}$ 利用乳酸为手性源, 发展了一 类柔性的 C2-对称的手性芳基碘化物，通式结构为图 11 中的结构 13. 作者在设计这一类手性芳基碘化物时, 既 考虑了碘化物制备的简单性，即三个片段之间通过简单 化学反应结合的问题. 也考虑了在形成的手性碘(III)中 间体中，如何营造好的手性环境. 他们认为三价碘与两 个羰基氧之间形成的 $n-\sigma *$ 相互作用以及碘(III)上的配体 与芳基碘化物之间形成的氢键相互作用, 为反应提供了 很好的手性环境，从而产生高对映选择性. 这类芳基碘 化物作为催化剂与 $m-\mathrm{CPBA}$ 共同作用, 在萗酚的不对称 去芳构化螺环内酯化反应中，产物可以达到 $90 \% \mathrm{ee}$ 左 右，通过简单重结晶，可以获得 $99 \% e e$ 值(图 11). 而采 用 Fujita 发展的单取代的芳基碘，对映选择性只有 $30 \%$ $e e$ 左右. 但这一反应收率受芳环取代基影响较大，仍然 存在较大的底物局限性. 特别是带有给电子基团如甲氧 基时, 收率较低, 可能是由于底物被氧化为二醌等副反 应导致.

此前所发展的手性高价碘促进的不对称反应，与过 渡金属促进的反应相比，对映选择性都相对较低，因而 实用性受到很大限制. 而 Ishihara 发展的这一类基于乳 酸的手性芳基碘化物，真正展现了手性有机碘化物在不 对称合成中隐藏的巨大的潜力. 因此也引起了广泛的关 注，不少课题组发展了基于乳酸结构的手性碘(I/III)化 合物，并应用到多种类型的不对称转化.

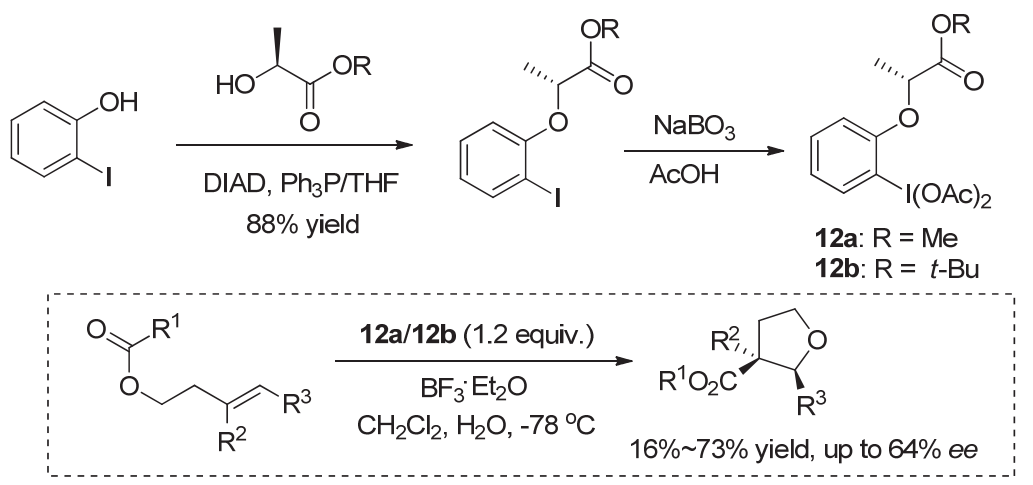

图 10 基于乳酸的手性高价碘试剂及烯烃的分子内不对称环化反应

Figure 10 Asymmetric intramolecular cyclization of alkene by lactic acid-based chiral hypervalent iodine reagents 


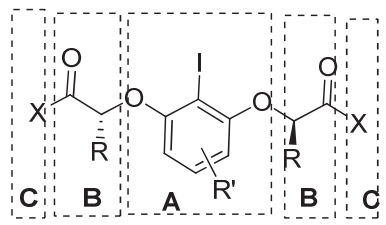

13

13a: $X=O E t, 13 b: X=O H$

13c: $X=\mathrm{NH}_{2}, 13 \mathrm{~d}: X=\mathrm{NHPh}$

13e: $X=\mathrm{NH}\left[3,5-\left(\mathrm{CF}_{3}\right)_{2} \mathrm{C}_{6} \mathrm{H}_{3}\right]$

13f: $X=\mathrm{NH}\left(3,5-{ }^{t} \mathrm{Bu}_{2} \mathrm{C}_{6} \mathrm{H}_{3}\right)$

13g: $X=$ NHMes, 13h: $X=N-p y r r$

13i: $X=\mathrm{NPh}_{2}$

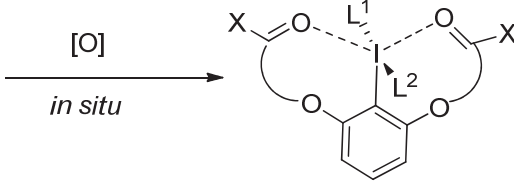

(X = YH or YR")

13'-I

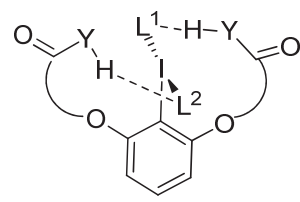

$(\mathrm{X}=\mathrm{YH})$

13 '-II

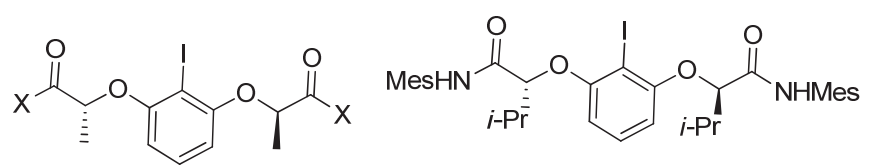

13a 13i

13j

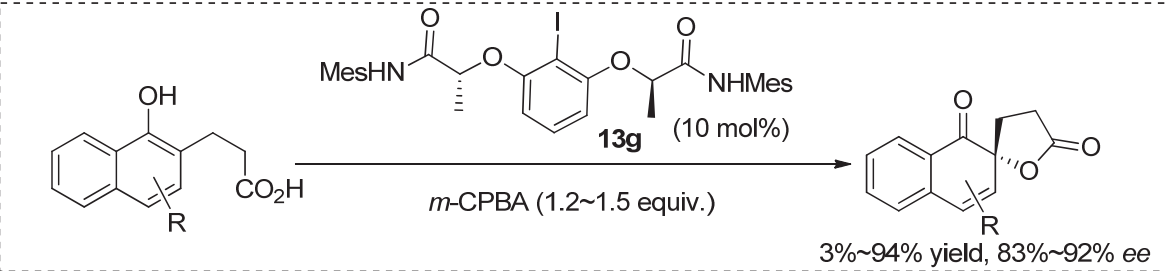

图 11 Ishihara 组发展的 C2 对称的柔性骨架芳基碘及 Kita 氧化去芳构螺环化反应研究

Figure 11 Lactic acid-based C2-symmetric chiral hypervalent iodine reagents by Ishihara et al. and the applications in enantioselective Kita oxidative spirocyclization

Fujita 等 ${ }^{[19 \sim 21]}$ 在他们原来单取代的芳基碘(III) 12a/12b 的基础上, 进行进一步的改进. 例如在碘的另 一个邻位引入取代基, 形成 2,6-位双取代的手性碘(III) 试剂, 包括 C2 对称的手性碘(III)试剂 14 等. 他们运用 这一类手性碘(III)试剂, 在多种类型的烯烃双官能团化 反应中, 取得了重要的进展(图 12). 例如: 2010 年, 他 们 ${ }^{[19]}$ 报道了邻羧基苯乙烯类化合物的内酯环化-对甲苯
磺酸酯化反应，对映选择性最高达到 $97 \%$ ee. 2011 年, 他们 ${ }^{[20]}$ 在烯烃的不对称分子间双官能团化反应中，产 物对映选择性高达 $96 \%$ ee . 2016 年, 在烯烃的氧化环化 芳基化反应中，实现了高对映选择性地构建复杂的并杂 环体系 ${ }^{[21 a]}$. 但在这些转化中, 由于多种副反应的存在, 大多情况下，都只能得到中等的收率.
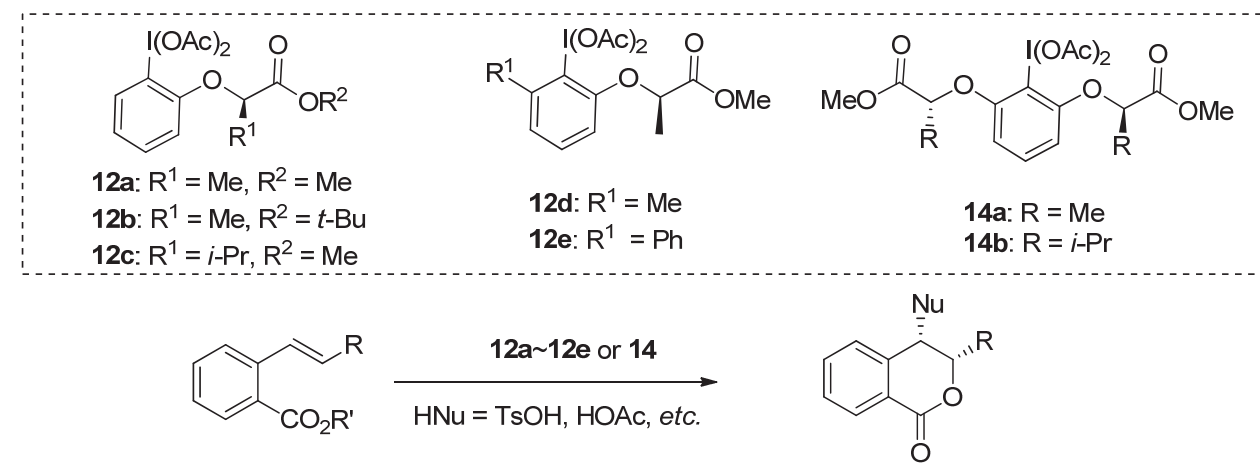

$$
57 \% \sim 84 \% \text { yield, up to } 97 \% \text { ee }
$$
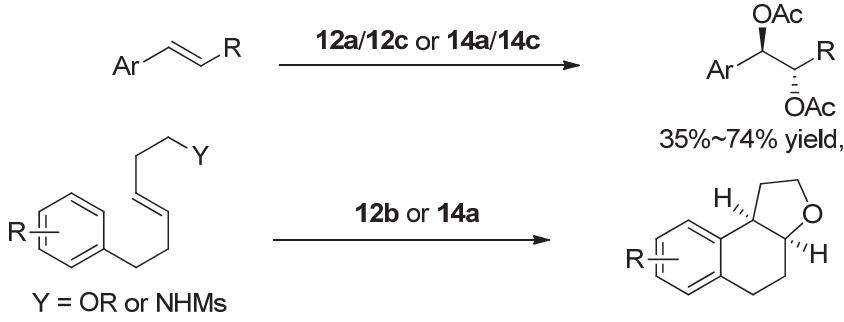

$35 \% \sim 74 \%$ yield, up to $96 \%$ ee
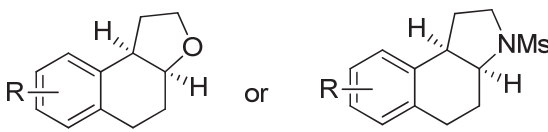

$17 \% \sim 88 \%$ yield, up to $95 \%$ ee $61 \% \sim 85 \%$ yield, $60 \% \sim 80 \%$ ee

图 12 Fujita 组在烯烃不对称双官能团化研究中的进展

Figure 12 Asymmetric bifunctionalization of alkenes by Fujita et al. 
Muñiz 等 ${ }^{[22 ~ 24]}$ 采用这类 $\mathrm{C} 2$ 对称的芳基碘(I/III)试 剂，高效地实现了一系列烯烃的双官能团化反应. 2011 年, 他们 ${ }^{[22]}$ 采用化学计量的芳基碘(III)试剂 14a, 高对 映选择性地实现了苯乙烯类化合物的双胺化反应, ee 值 最高达到 $95 \%$. 对于苯丙烯, 反应生成两个手性中心, 同样可以获得高对映选择性. 但对于烷基烯烃, 反应几 乎没有对映选择性(图 13a). 2017 年, 他们 ${ }^{[23]}$ 进一步改进 这类 C2 对称的芳基碘, 采用催化量的芳基碘(I)化合物 15a, 用 $m$-CPBA 作为氧化剂, 实现了高对映选择性的 不对称催化双胺化反应, 对于苯乙烯和苯丙烯类底物, 产物均获得高达 $98 \% \mathrm{ee}$, 但大多情况下只有中等收率 (图 13b). 此外, 2016 年, 他们 ${ }^{[24]}$ 还采用催化量的芳基碘 (I) 化物 $15 \mathrm{~b}$ 以及 16 , 实现了苯乙烯类化合物的高对映选 择性双乙酸酯化反应, 产物收率中等到良好, 对映选择 性最高达到 94\% ee(图 13c).

2012 年, Wirth 等 ${ }^{[25]}$ 同样利用这类有机碘(III)试剂 17, 实现了烯烃分子内的不对称 $N, O$ 双官能团化成环 反应, 生成具有四取代的并环手性中心产物, ee 值最高 可以达到 $99 \%$, 但仅限于分析量的小反应. 在更大量的 反应中, 反应收率在大多情况下不高, 并且对映选择性 有所降低, 但仍然可以达到 $90 \%$ ee 以上(图 14a). 最近,
Masson 等 ${ }^{[26]}$ 采用 Ishihara 的催化剂 13g, 在烯烃不对称 内酯环化反应中, 也取得了中等程度的收率以及较好的 对映选择性，最高达到 94\%ee(图 14b).

2013 年, Nevado 等则使用这类骨架发展了氧化的 氟试剂, 代表性结构为化合物 18a. 他们 ${ }^{[27 a]}$ 采用手性芳 基碘(III)化合物 18a, 首次发展了非活化烯烃的分子内 高对映选择性不对称胺基化氟化反应，以中等到良好的 收率和对映选择性生成手性哌啶以及高哌啶产物. 通过 简单重结晶, 可以获得 $99 \% e e$ (图 15a). 他们在苯乙烯 类化合物的分子间胺基化氟化反应中，同样可以高效率 地得到 2-氟-2-芳基乙胺类化合物，但遗憾的是，未能实 现不对称反应.

最近, Falivene 和 Rueping 等 ${ }^{[27 b]}$ 在研究基于茚酮结 构的 $\beta$-酮酯化合物的不对称氟化反应时, 同样利用了 Ishihara 等发展的这类骨架, 发展了芳基碘化物 $\mathbf{1 8 b}$, 可 以中等的收率和较高的对映选择性实现不对称氟代反 应, 反应可以在室温和空气中进行, 产物 $e e$ 值大多在 $70 \%$ ～92\%之间(图 15b). 他们对反应机理及过渡态进行 了计算，对酯基部位、甲基部位以及芳环上的取代基等 都进行了探讨.
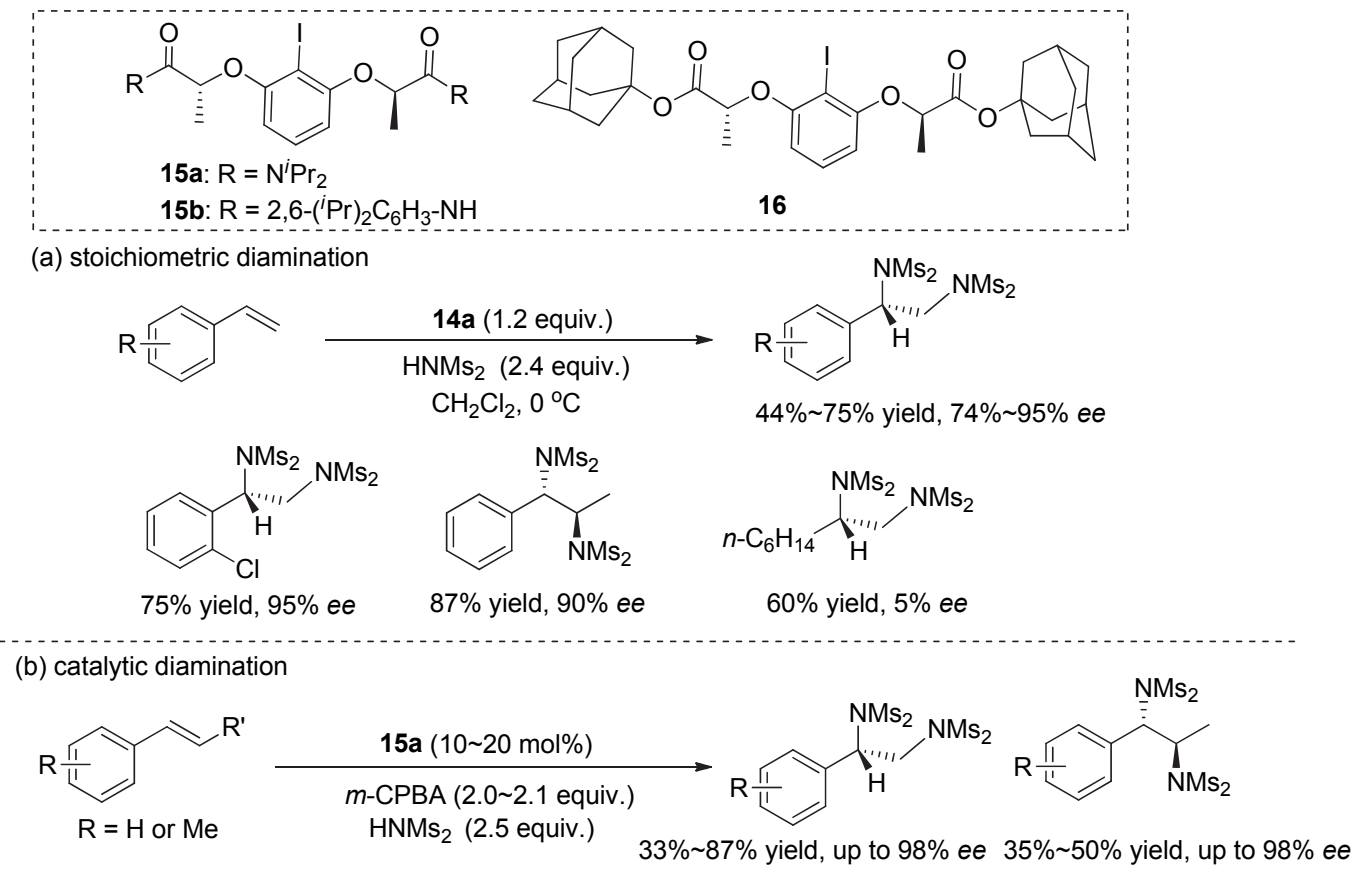

(c) catalytic diacetoxylation

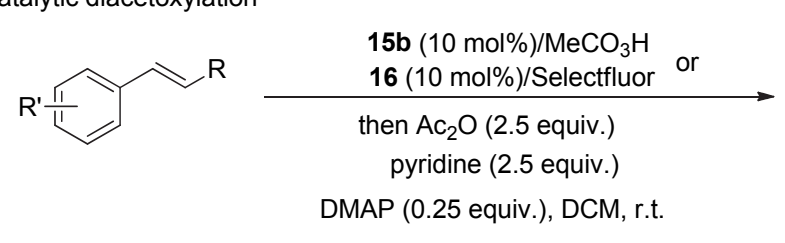

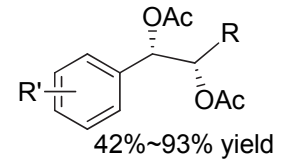

with 15b: $\mathrm{R}=\mathrm{H}, 63 \% \sim 94 \%$ ee with 16: $\mathrm{R}=\mathrm{H}, 62 \% \sim 88 \%$ ee $\mathrm{R}=\mathrm{CH}_{2} \mathrm{OAc}, 78 \% \sim 95 \%$ ee

图 13 Muñiz 组关于苯乙烯类化合物的不对称双官能团化研究

Figure 13 Asymmetric bifunctionalization of styrenes by Muñiz et al. 
(a) Wirth: Stereoselective oxyaminations

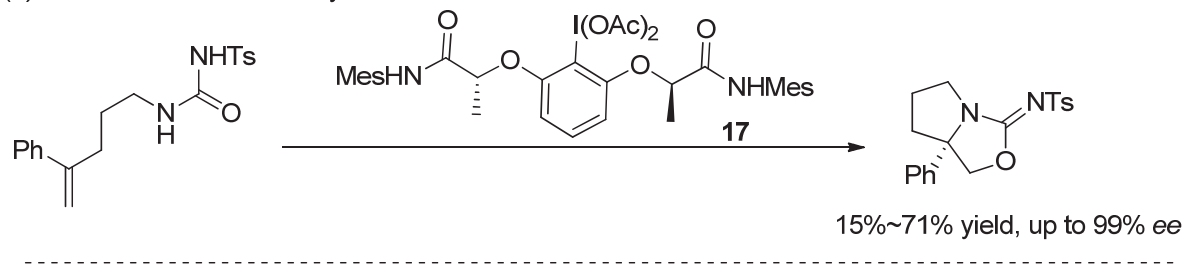

(b) Masson: enantioselective sulfonyl- and phosphoryl-oxylactonizations

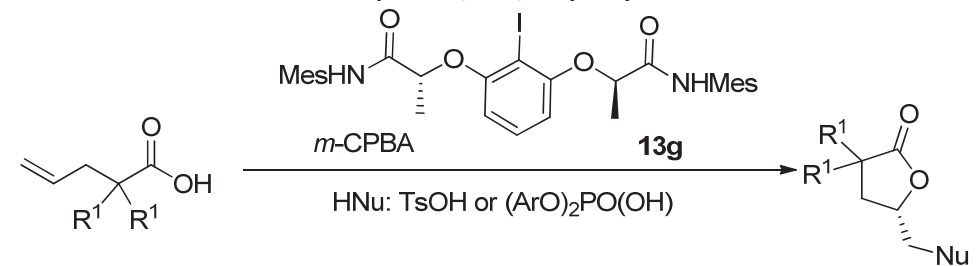

Sulfonyloxylactonization: $25 \% \sim 76 \%$ yield, $58 \% \sim 81 \%$ ee

Phosphoryl-oxylactonization: 51\% 88\% yield, 70\% 94\% ee

图 14 其它的烯烃不对称双官能团化反应

Figure 14 Other examples for asymmetric bifunctionalization of alkenes

(a) Regio- and enantioselective aminofluorination of alkenes

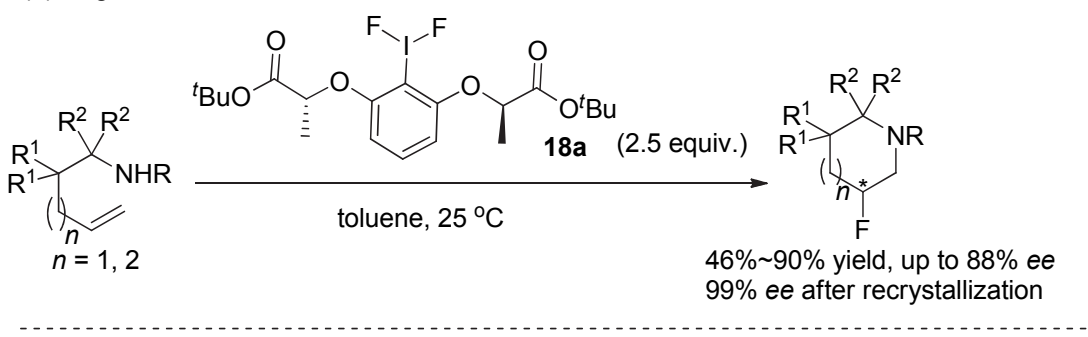

(b) Asymmetric fluorination of keto esters

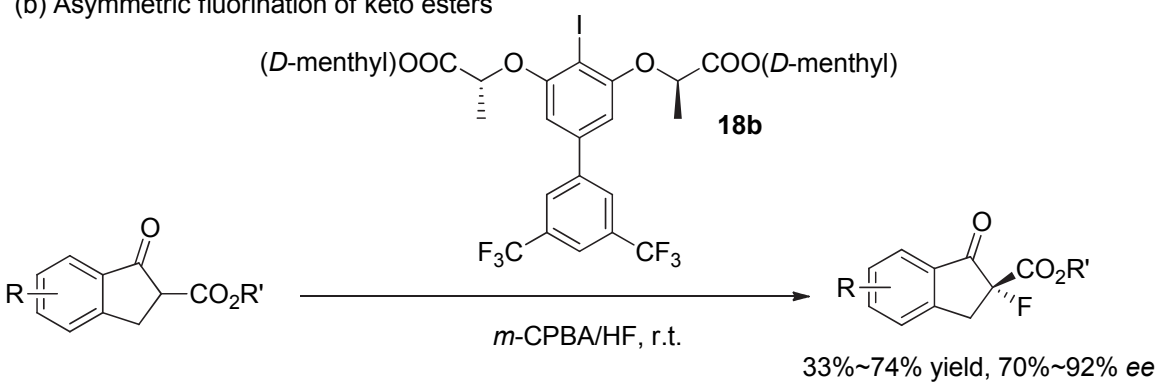

图 15 手性碘氟化试剂及不对称氟化反应

Figure 15 Chiral aryliodine (I/III) reagents for enantioselective fluorinations

特别值得注意的是, 随着 Ishihara 类手性碘(I/III)化 合物的广泛应用, 新的催化剂以及新的不对称反应类型 也有了发展. 以往基于乳酸酰胺所发展的 Ishihara 类型 手性高价碘试剂, 大多为二级酰胺, 可以通过酰胺氮上 的氢原子与底物或溶剂等形成氢键，从而调控反应的对 映选择性. 但在 2014 年, 龚流柱等 ${ }^{[28]}$ 研究催化的不对 称氧化 $\mathrm{C}-\mathrm{C}$ 偶联反应时, 发现使用三级乳酸酰胺芳基 碘化物, 效果远比二级酰胺芳基碘化物要好. 其中采用 脯氨酸的三级乳酸酰胺芳基碘化物 19 作为催化剂, $m$-CPBA 作为氧化剂时, 效果最佳. 反应产物可以获得 较高的对映选择性, ee 值最高达到 $90 \%$. 从而实现了螺 二氧化吲哚类化合物的高对映选择性构建. 但由于副反 应等存在, 大多情况下收率只能达到中等(图 16a). 2015 年, 他们 ${ }^{[29]}$ 又采用二级酰胺的有机碘化物 13g, 实
现了菜酚的不对称去芳构化反应构建手性螺环结构, 产 物收率中等到良好, 对映选择性最高达到 $92 \% \mathrm{ee}$ (图 16b). 2016 年, 杜云飞等 ${ }^{[30]}$ 采用和龚流柱同样的手性芳 基碘化物 19, 应用于串联氧化的 $\mathrm{C}-\mathrm{O} / \mathrm{C}-\mathrm{C}$ 键形成, 实现了高对映选择性构建氧化吲哚类的螺环化合物, 产 物基本在 80\% 90\% ee 左右(图 16c).

除了氧化的交叉偶联反应，这类乳酸的手性芳基碘 (I/III)试剂, 在新的不对称氧化重排反应中也取得了突 破性进展. 2013 年, Wirth 等 ${ }^{[31]}$ 首次报道了手性高价碘 (III)促进的 $\beta$-芳基- $\alpha, \beta$-不饱和酮的高对映选择性氧化重 排反应，生成 $\alpha$-芳基酮类化合物，最高可达 $99 \%$ ee . 2016 年, 他们 ${ }^{[32]}$ 进一步针对 1,1-双取代烯烃发展了不对 称氧化重排反应，生成手性 $\alpha$-芳基酮，大多数情况下， 都获得了中等到良好的收率和对映选择性(图 17). 
(a) oxidative cross-coupling

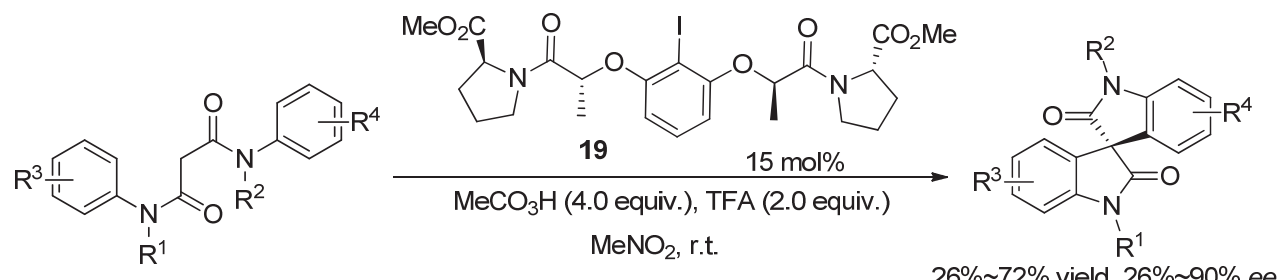

(b) oxidative dearomatizative spirocyclization
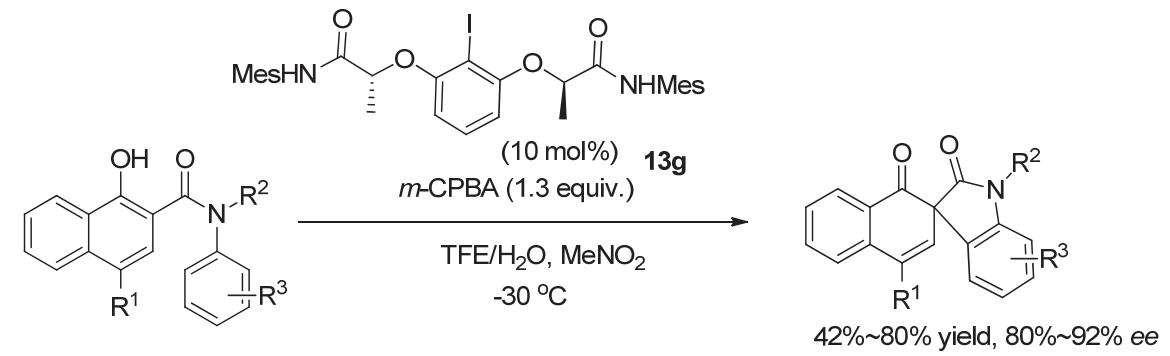

(c) oxidative $\mathrm{C}-\mathrm{C} / \mathrm{C}-\mathrm{O}$ cross-coupling
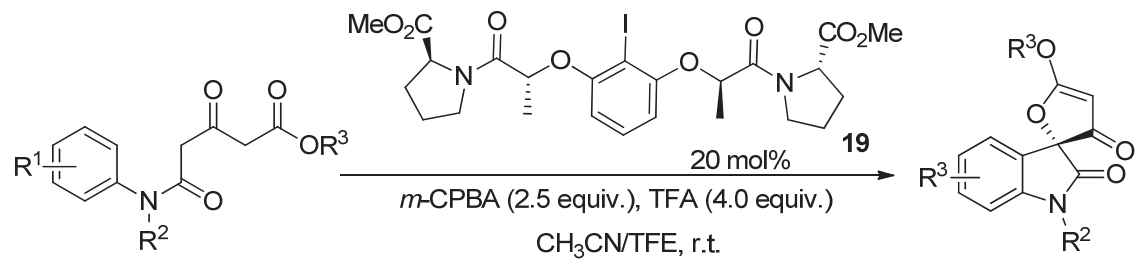

$26 \% \sim 82 \%$ yield, $74 \% \sim 91 \%$ ee

图 16 不对称氧化交叉偶联反应构建螺环结构

Figure 16 Asymmetric oxidative coupling for the formation of spirocycles

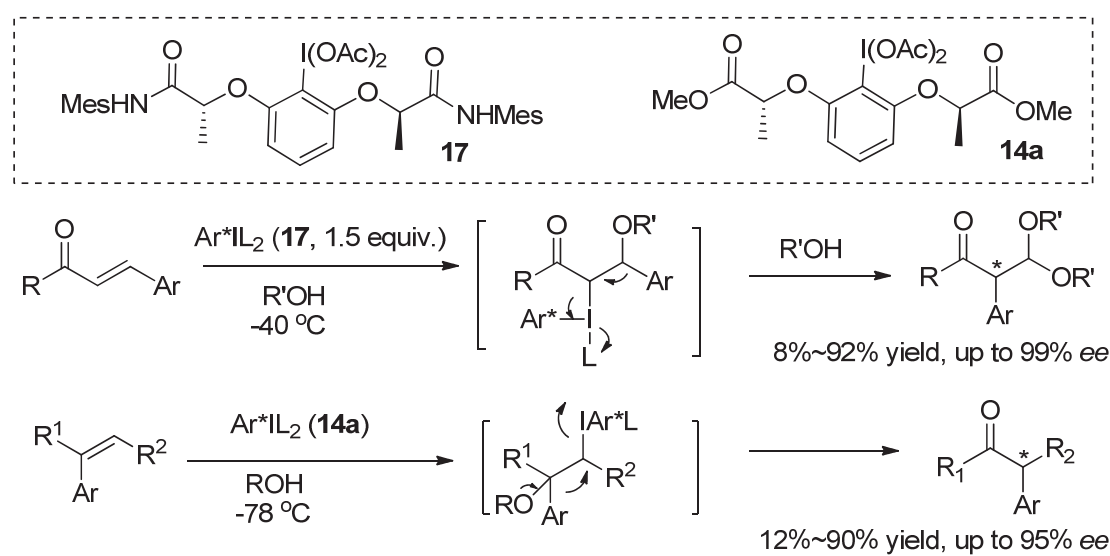

图 17 Wirth 组对烯烃的不对称氧化重排反应研究

Figure 17 Asymmetric oxidative rearrangements by Wirth et al.

而在 2016 年, Jacobsen 等 ${ }^{[33]}$ 在使用 20a/20b 研究芳 基碘化物催化烯烃的二氟化反应时, 发现当控制温度在 $4{ }^{\circ} \mathrm{C}$ 时, $\alpha, \beta$-不饱和酰胺可以高立体选择性生成 $1,2-二$ 氟化产物(图 18a). 而当反应温度控制在 $-40{ }^{\circ} \mathrm{C}$ 时, $\alpha, \beta$ 不饱和酰胺或酯底物的催化二氟化反应发生了氧化重 排生成含二氟甲基手性中心的化合物(图 18b), 产物大 多获得高对映选择性, 最高达到 $96 \% e e^{[34]}$. 使用未活化
的普通烯烃时，同样可以获得中等到良好的对映选择 性. 他们所使用的芳基碘化物 $\mathbf{2 0 a} / \mathbf{2 0 b}$, 同样是根据 Ishihara 等的芳基碘化物改进而来，在手性中心位置引 入位阻更大的苄基取代甲基，可以获得高对映选择性. 同时把酯基部分改为茮酯，可以提高反应活性，使反应 可以在低温下进行, 从而提升对映选择性. 最近, Houk 和薛小松等 ${ }^{[35]}$ 对这一反应的化学选择性和立体选择性 
进行了计算, 进一步明确了这类重排反应对映选择性的 机理.

最近，Jacobsen 等 $^{[36]}$ 又利用这类芳基碘(I)化合物， 实现了烯烃的催化不对称氟化/胺基化反应, 可以高对 映选择性构建带两个手性中心的含氟氮杂三元环等化 合物, 产物只有单一立体异构体, 对映选择性非常优异, 大多在 $90 \%$ ee 以上, 最高可以达到 $97 \%$ ee (图 19).

Jacobsen 的工作中, 对于手性碘的改造非常有意思, 他们不仅对乳酸酯部分进行了改造, 以期改进反应活性 和对映选择性. 同时, 他们在芳环上加入了吸电子的酯 基，尽管他们并未说明这一基团的加入对于反应的影 响, 但吸电子基团的加入, 对于碘化物的电性显然有所 影响, 值得进一步研究.

2017 年, Wirth 等 ${ }^{[37]}$ 在芳环上引入不同的取代基, 如给电子的甲基以及吸电子的三氟甲基, 发展了一系列
新结构的手性芳基碘(I/III)试剂 $21 \sim 22$, 来研究高价碘 试剂中芳环的电性对于氧化重排反应的活性以及对映 选择性的影响(图 20). 从他们的研究结果来看, 加入给 电子基或吸电子基对于氧化重排反应的活性和对映选 择性没有明显的影响. 但这一结果在其它手性高价碘促 进的反应中，并不一定完全符合. 手性碘试剂的电性对 于反应活性以及对映选择性的影响, 在研究中仍然是一 个非常值得思考和深入研究的方面.

在最近的一个报道中, Maruoka 等 ${ }^{[38]}$ 采用手性䒢醇 为骨架，发展芳基碘化物，用于酚的不对称氧化去芳构 化. 最终, 为了获得高的对映选择性, 他们仍然选择在 碘的另一个邻位引入了手性乳酸骨架, 代表性结构为 23, 才能获得高的对映选择性, 但仍然只能达到中等收 率(图 21).

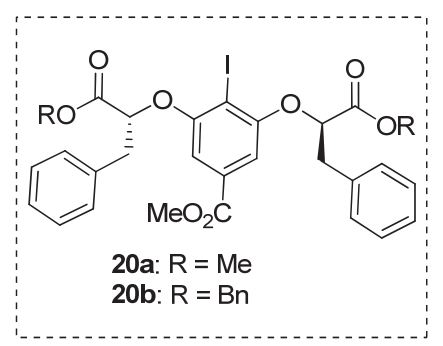

(a) Catalytic and diastereoselective 1,2-difluorination of alkenes

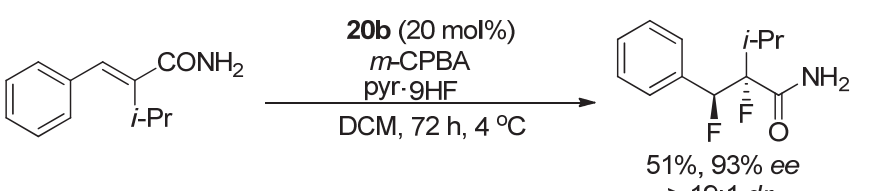

(b) asymmetric difluorination via oxidative rearrangement

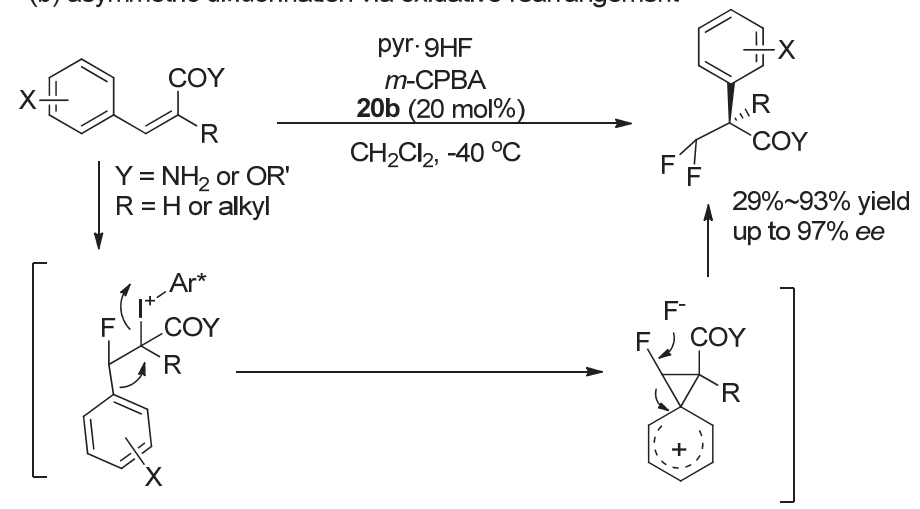

图 18 烯烃不对称 1,2-二氟化及氧化重排反应

Figure 18 Diastereoselective 1,2-difluorination and oxidative rearrangement of alkenes by Jacobsen et al.

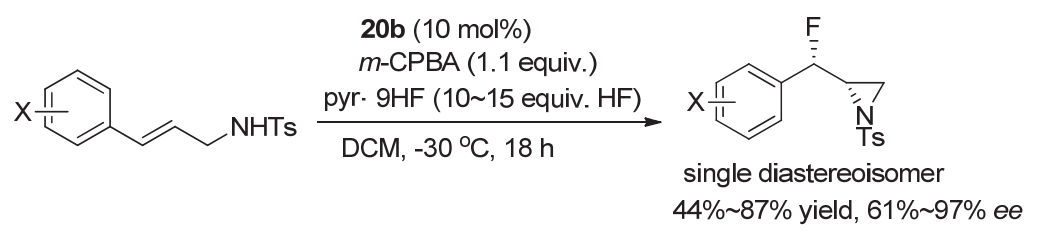

图 19 烯烃的催化不对称氟化/胺基化

Figure 19 Catalytic diastereo- and enantioselective fluoroamination of alkenes 
<smiles>[X]C(=O)C(C)Oc1cc([R])cc(O[C@H](C)C([X])=O)c1I</smiles>

21a: $R=\mathrm{Me}, \mathrm{X}=\mathrm{OMe}$

21b: $\mathrm{R}=\mathrm{CF}_{3}, \mathrm{X}=\mathrm{OMe}$

21c: $\left.\mathrm{R}=\mathrm{CF}_{3}, \mathrm{X}=2,6-{ }^{i} \mathrm{Pr}\right)_{2} \mathrm{C}_{6} \mathrm{H}_{3}-\mathrm{NH}$

21d: $\mathrm{R}=\mathrm{CF}_{3}, \mathrm{X}=2,4,6-\mathrm{Me}_{3} \mathrm{C}_{6} \mathrm{H}_{2}-\mathrm{NH}$<smiles>[X]C(=O)C(C)Oc1cc([R])cc(O[C@H](C)C([X])=O)c1I(OC(C)=O)OC(C)=O</smiles>

22a: $R=M e, X=O M e$

22b: $\mathrm{R}=\mathrm{CF}_{3}, \mathrm{X}=\mathrm{OMe}$

22c: $\mathrm{R}=\mathrm{CF}_{3}, \mathrm{X}=2,6-{ }^{\prime}(\mathrm{Pr})_{2} \mathrm{C}_{6} \mathrm{H}_{3}-\mathrm{NH}$

22d: $\mathrm{R}=\mathrm{CF}_{3}, \mathrm{X}=2,4,6-\mathrm{Me}_{3} \mathrm{C}_{6} \mathrm{H}_{2}-\mathrm{NH}$

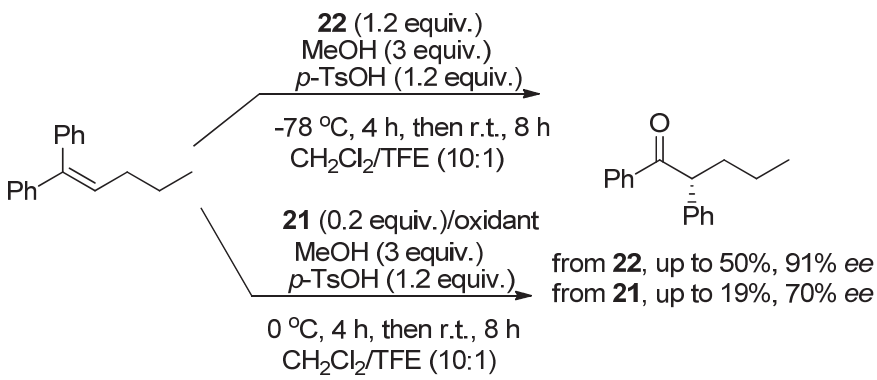

图 20 芳基碘(I/III)试剂的电性对于氧化重排反应活性和对映选择性的影响研究

Figure 20 The influences of electron property of chiral aryliodine reagents to the reactivity and enantioselectivity in oxidative rearrangement reactions<smiles>[R]c1cc(OCCO)c([R])cc1O</smiles><smiles>[R]c1cc([R])c(OC([3H])([3H])[3H])cc1[R]</smiles>

$\mathrm{H}_{2} \mathrm{O}$<smiles>[R]C1=CC([R])(O)C([R])=CC1=O</smiles>

$34 \% \sim 73 \%$ yield, $63 \% \sim 84 \%$ ee

$$
\text { C }
$$

$\mathrm{Ar}=2,6-\mathrm{Ph}_{2} \mathrm{C}_{6} \mathrm{H}_{3}$

23

图 21 Maruoka 发展的手性芳基碘化物

Figure 21 Chiral iodoarenes by Maruoka et al.

以乳酸为手性源发展的这一类手性芳基碘化合物, 经过多个课题组的发展, 在多种反应类型中, 都展现出 比其它高价碘试剂或催化剂更好的效果, 目前, 对这类 芳基碘化物的研究还在继续深入, 可以预见, 将会有更 多激动人心的报道出现.

\subsection{3 基于 “手性池” 中氨基醇等结构发展的其它类型 手性芳基碘}

基于柔性骨架中心手性的高价碘试剂或前体，除了 基于 Koser 类的结构(6 11) 以及 Fujita 和 Ishihara 发展 的乳酸类芳基碘结构外, 其它类型的结构也非常多. 各 类手性片段, 大多来源于 “手性池” 中的结构, 如氨基 酸、氨基醇等, 通过成酯、酰胺等方式, 与芳基碘片段 连接, 发展了多种类型的高价碘试剂或前体(图 22).

例如, 2000 年, Zhdankin 等 ${ }^{[39]}$ 报道了一类手性芳基 碤(V)试剂 24, 在这一结构中, 手性片段来源于 “手性 池” 中的氨基酸分子. 这类手性高价碘结构, 在硫醚的 不对称氧化中, 表现并不好, $e e$ 值仅有 $11 \% \sim 16 \%$. 而他
们 ${ }^{[40]}$ 在 2006 年又报道了另一种手性芳基碘(V)试剂 25, 手性元素来源于 “手性池” 中的脯氨酸. 作者认为分子 中的碘原子与来自两个酰胺基团的氧原子之间可能存 在假键合或配位，这将允许形成七元环，从而有可能实 现较好的手性控制. 但这一试剂在硫醚的不对称氧化 中, 同样表现不佳, 仅有 $29 \%$ ee.

Wirth 等 ${ }^{[41]}$ 报道了一系列酯类的芳基碘化物，代表 性结构如 26 和 27. 他们 ${ }^{[22]}$ 使用 27 在苯丙酮的 $\alpha$-位不对 称对甲苯磺酸酯化反应中, 最高获得 $26 \% e e$, 而在 5-羰 基酸的羰基 $\alpha$-位内酯化反应中, 则几乎没有观察到对映 选择性.

2013 年, Harned 等 ${ }^{[43]}$ 基于 Ishihara 类碘化物的特点, 采用酒石酸为手性源, 发展了另一类的手性芳基碘化 物, 代表性结构为化合物 28. 应用于酚类化合物的不对 称去芳构化, 但收率和对映选择性并不令人满意(图 23).

氨基醇作为手性源也被用于各类手性芳基碘化物 中. 2009 年, Birman 等 ${ }^{[4]}$ 发展了与 Zhdankin 类似的手性 
<smiles></smiles>

24 Zhdankin 2000<smiles>[R]C(=O)[C@H]1CCCN1C(=O)O[V](=O)(=O)c1ccccc1</smiles>

25a/25b: R = Me, 4-Br-benzyl Zhdankin 2006<smiles>CC1CCC(C(C)C)C(OC(=O)Cc2ccccc2I)C1</smiles>

26 Wirth 2008

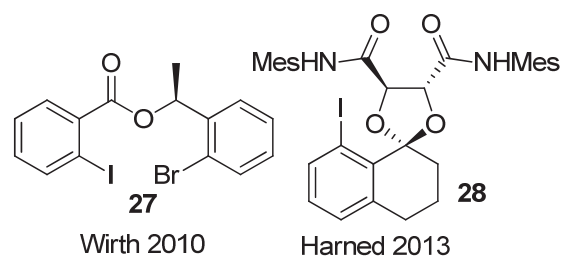

Wirth 2010<smiles>Cc1cccc(C(=O)NC(C)C(O)c2ccccc2)c1I</smiles>

31<smiles>CC(NC(=O)Cc1ccccc1I)C(O)c1ccccc1</smiles>

32

Birman 2009

Legault 2012<smiles>CC(=O)NC[C@H](C)Oc1cccc(O[C@H](C)CNC(C)=O)c1I</smiles>

Ishihara 2013<smiles>Cc1cc(Cl)cc(C2=NCC(c3ccccc3)O2)c1I</smiles><smiles>CC(=O)NCC(C)Oc1cccc(OCCNC(=O)OC(C)(C)C)c1I</smiles>

Ciufolini 2017

图 22 基于柔性骨架中心手性的其它高价碘试剂或前体

Figure 22 Other chiral hypervalent reagents or precursors based on conformationally flexible skeletons with central chirality

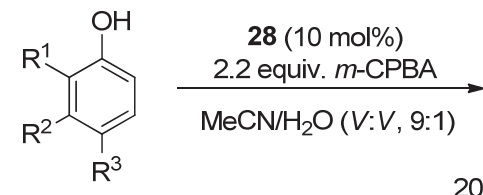

$20 \% \sim 94 \%$ yield, $<60 \%$ ee

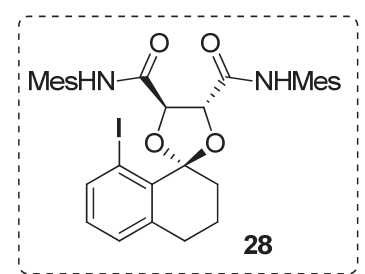

图 23 Harned 发展的手性芳基碘及不对称去芳构化反应研究

Figure 23 Chiral iodoarenes by Harned et al.

碘(V)试剂. 手性元素来源于 “手性池” 中的氨基醇，通 过转化生成邻位带手性噁唑啉取代基的芳基碘(V)化合 物, 代表性化合物为 29. 这类手性碘(V)试剂被用于邻 位烷基酚的不对称去芳构化/[4+2]D-A 二聚反应，反应 收率很好, 但令人遗憾的是, 只有中等程度的对映选择 性(图 24).

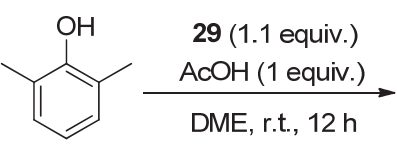

图 24 酚的不对称去芳构/[4+2]串联反应

Figure 24 Asymmetric dearomatization/[4+2] tandem reactions of phenols

2012 年, Legault 等 ${ }^{[45]}$ 报道了以类似的邻位带手性 噁唑啉取代基的芳基碘化物作为催化剂, 代表性结构为 30. 他们用碘化物 30 为催化剂, 使用 $m$-CPBA 作为氧化 剂, 实现了羰基化合物 $\alpha$-位的对甲苯磺酸酯化反应, 但 对映选择性也仅在 $50 \%$ ee 左右.
而几乎同时由 Moran 等 ${ }^{[46]}$ 报道了采用手性氨基醇 作为手性源的类似化合物 31 和 32, 在 $m$-CPBA 作为氧 化剂, 化合物 31 催化苯丙酮的羰基 $\alpha$-位对甲苯磺酰化 反应中, 同样表现不佳, 对映选择性较低, 仅有 $18 \%$ ee. 但在分子内形成五元环的反应中，采用手性碘化物 32 为催化剂, 取得了中等的对映选择性, 最高达到 $51 \%$ $e e$, 是这类不对称内酯环化反应中最好的结果(图 25).

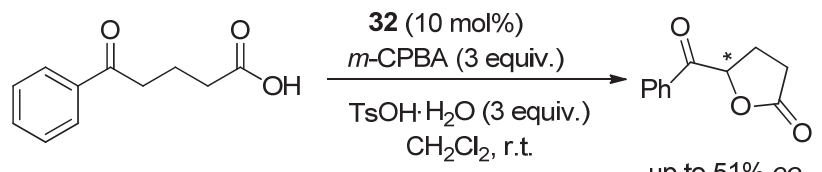

图 25 羰基 $\alpha$-位的分子内不对称内酯环化反应

Figure 25 Asymmetric lactonization of 5-oxo-5-phenylpentanoic acid

Fujita 和 Ishihara 基于手性乳酸结构发展的手性芳 基碘(I/III)试剂在不对称合成中得到了广泛的发展和应 用. 在多种类型的不对称转化中，展现出比其它类型的 手性碘试剂更好的对映选择性, 为手性高价碘化学打开 了一扇大门. 但值得注意的是, 他们 2010 年发展的这类 手性芳基碘，尽管在 1-荎酚的不对称 Kita 氧化去芳构螺 环化反应中取得了很好的对映选择性，但对于 2-萗酚以 
及苯酚类化合物的去芳构化反应，其反应活性及对映选 择性都不高. 因此在他们 2010 年工作的基础上, 2013 年, Ishihara 等 ${ }^{[47]}$ 采用氨基醇为手性源, 又进一步发展了 第二代 C2 对称的柔性手性芳基碘化物, 其代表性结构 为化合物 33. 在苯酚类化合物的不对称去芳构化螺环 内酯化反应中, 第一次实现了非常高的对映选择性, 最 高达到 99\% ee. 并且作为催化剂前体的芳基碘化物, 只 需要 $1 \sim 10 \mathrm{~mol} \%$, 转化效率也进一步提高. 他们提出了 该反应的作用模式, 提出了溶剂和氢键作用对反应对映 选择性以及转化效率的影响等(图 26a). 他们 ${ }^{[48,49]}$ 利用 这一类芳基碘, 实现了包括 $\beta$-菜酚、 $\alpha$-菜酚和苯酚等化 合物的多类不对称去芳构螺环化反应(图 26b), 都取得
了较好的收率以及高对映选择性，与他们发展的第一代 基于乳酸的手性芳基碘比较，往往更胜一筹.

最近, Ciufolini 等 ${ }^{[50]}$ 也利用类似的芳基碘化物 34, 与 $m-\mathrm{CPBA}$ 一起作用, 实现了其它类型的萗酚不对称去 芳构螺环化反应，取得了很高的对映选择性(图 27).

2016 年, Gilmour 等 ${ }^{[51]}$ 在研究苯乙烯类化合物的不 对称 1,2-二氟化反应时, 采用了 Ishihara 发展的第二代 芳基碘化物 33，但对映选择性不好，仅能获得约 $22 \%$ ee. 2018 年, 他们 ${ }^{[52]}$ 最终采用第一代乳酸类似的芳基碘 化物 35, 实现了较高对映选择性的 1,2-二氟化反应, 产 物最高可获得 $88 \%$ ee (图 28). 但反应中存在 1,1-二氟代 副产物，大多情况下只有中等收率.

(a)

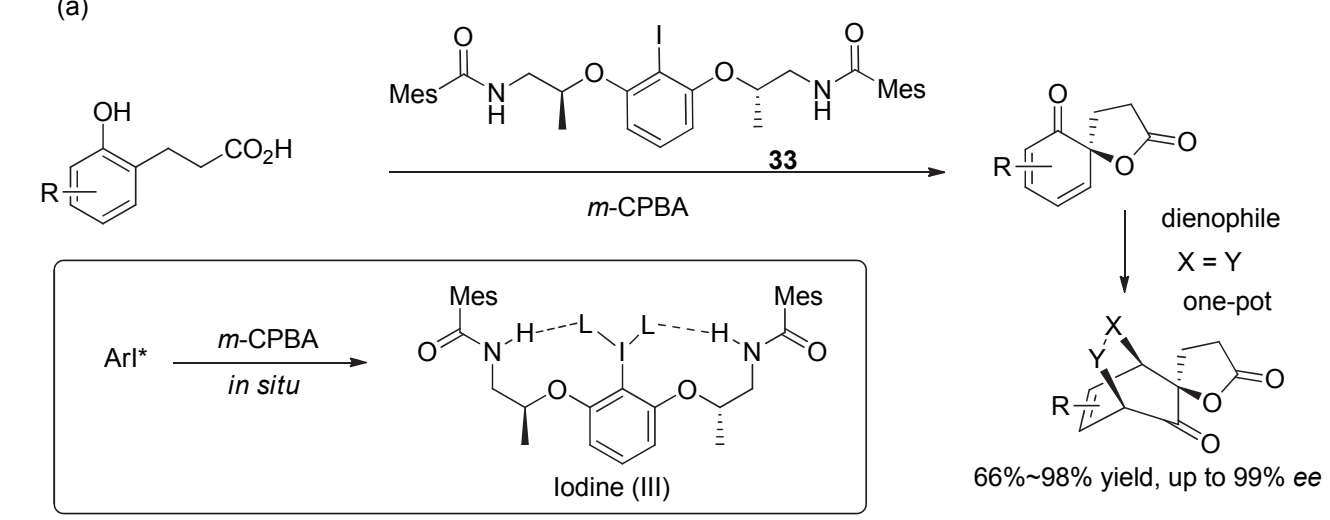

(b)

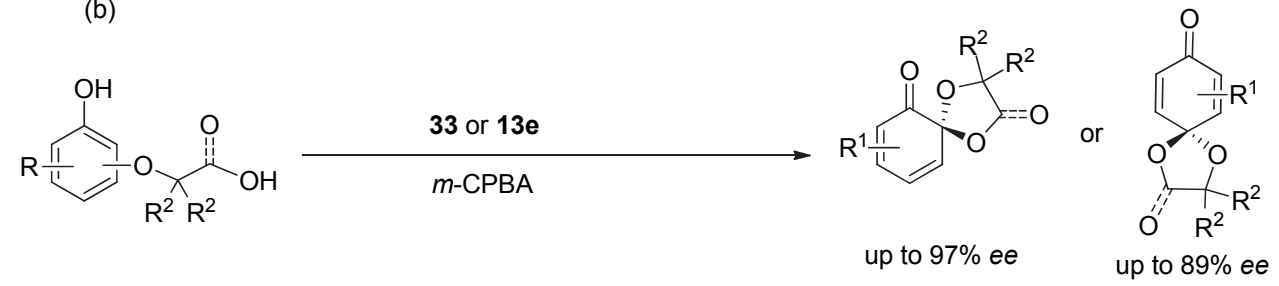

图 26 Ishihara 发展的第二代柔性结构 C2-对称的手性芳基碘

Figure 26 The second generation of $\mathrm{C} 2$-symmetric and conformationally flexible chiral iodoarenes by Ishihara

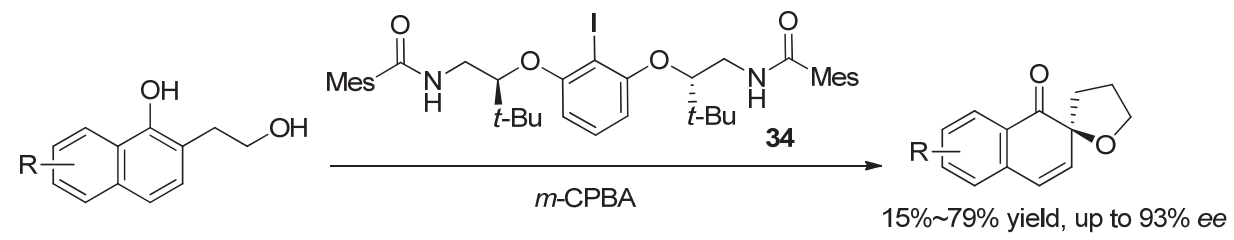

图 27 不对称去芳构螺环化反应研究

Figure 27 Asymmetric dearomatizing spirocyclization
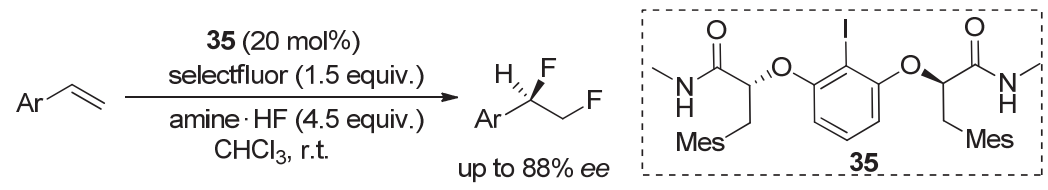

图 28 烯烃的 1,2-二氟化反应

Figure 28 1,2-difluorotination of alkenes 


\section{3 基于刚性骨架的手性芳基磑化物}

在前面我们所讨论的手性芳基碘化物或其氧化物, 其手性基本为中心手性, 大多来源于 “手性池” 中的各 类结构. 这些柔性结构的手性芳基碘化物, 在不对称转 化中得到了广泛的发展和应用.

除此之外，一些在过渡金属催化中常用的刚性手性 骨架, 也被用来发展新的手性高价碘化物或手性芳基碘 化物. 在这一节中, 我们将对此进行介绍.

2.3.1 基于手性联菜或联苯骨架发展的手性芳基碘试 剂或前体

在过渡金属催化的反应中, 手性联芸或联苯基团是 常用的一类骨架. 在手性芳基碘试剂发展中, 早在 1990 年, Ochiai 等 ${ }^{[53]}$ 采用联萗基团为手性骨架, 报道了第一 类带有轴手性结构的手性芳基碘(III)试剂, 代表性结构 为 36 37. 他们研究认为化合物构型稳定性不高.

此后, 采用轴手性联荥或联苯骨架发展的芳基碘试
剂, 在不对称合成中逐渐得以发展. 图 29 中列出了一系 列代表性的手性芳基碘试剂或前体，在多种类型的不对 称转化中得到了广泛发展及应用。

1999 年, Ochiai 等 ${ }^{[4]}$ 采用手性联䒺芳基碘做成二芳 基碘鎓盐 38, 首次用于环状 $\beta$-酮酯的不对称 $\alpha$-芳基化 反应. 反应的对映选择性达到中等，产物可以获得 $53 \%$ $e e$, 但收率较低(图 30).

2011 年，徐镇江和支志明等 ${ }^{[55}$ 也采用手性联䒺碘 (III) 化合物 39, 在烯烃的分子内氮杂环丙烷化反应中进 行尝试, 但效果欠佳, 对映选择性仅达到 16\% ee(图 31).

Quideau 等发展了一系列的联荎或联苯骨架的手性 芳基碘试剂，应用于酚的不对称去芳构化反应等(图 32). 2009 年, Quideau 等 ${ }^{[56]}$ 报道了以 $m$-CPBA 为共氧化剂, 与手性联䒺碘代芳烃 $\mathbf{4 0}$ 促进苯酚类化合物的不对称去 芳构化羟基化反应，产率高达 90\%，对映选择性最高可 达到 $50 \%$ ee. 2014 年他们 ${ }^{[57]}$ 又报道了联苯骨架的手性<smiles>[X]c1ccc2ccccc2c1-c1c([R])ccc2ccccc12</smiles>

36: $\mathrm{X}=\mathrm{I}$ or $\mathrm{I}(\mathrm{OAc})_{2}$ $\mathrm{R}=\mathrm{H}$ or $\mathrm{X} \quad$ Ochiai 1990<smiles>CC(=O)OI(OC(C)=O)c1ccc2ccccc2c1-c1c(I)ccc2ccccc12</smiles>

37<smiles>[R]c1ccc2ccccc2c1-c1c([PH+](F)(F)c2ccccc2)ccc2ccccc12</smiles>

Ochiai 1999<smiles>Oc1ccc2ccccc2c1-c1c(O)ccc2ccccc12</smiles>

Xu \& Zhi 2011<smiles>COc1ccc2ccccc2c1-c1c(I)c(C(=O)O)cc2ccccc12</smiles>

Quideau 2009<smiles>COC(=O)c1c2c(cc(C(C)=O)c1C1=C([O-])C(C(C)=O)=CC3CCCCC13)CCCC2</smiles>

Quideau 2014

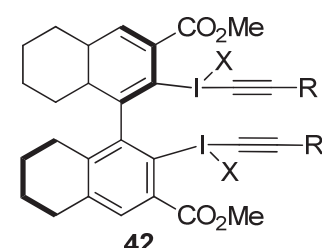

Quideau 2017<smiles>CCOOC1C(=O)N(Oc2c(I)cc3ccccc3c2-c2cc3ccccc3cc2I)C(=O)C1OCc1ccccc1</smiles>

2013 Berthiol \& Einhorn

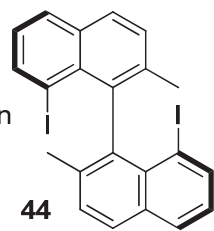

Dohi \& Kita 2017

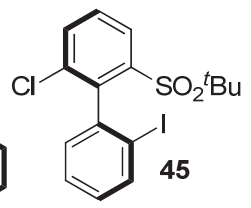

Masson 2017

图 29 基于手性联菜或联苯骨架发展的芳基碘试剂和前体

Figure 29 Chiral aryliodine reagents or precursors based on chiral binaphthalene or biphenyl skeletons

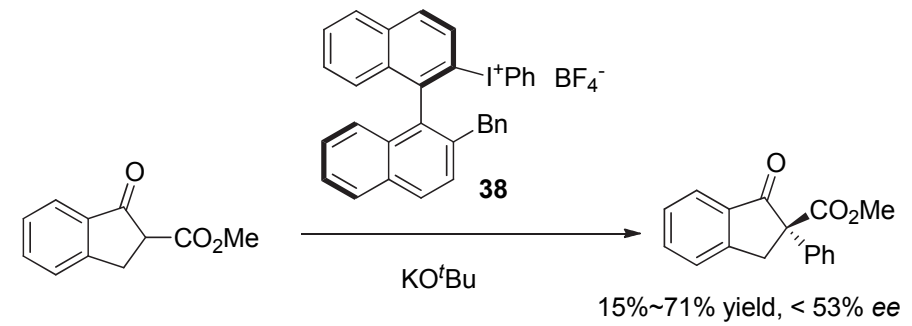

图 30 手性碘鎓盐在羰基 $\alpha$-位芳基化反应中的研究

Figure 30 Chiral diaryliodonium salts for asymmetric $\alpha$-arylation of $\beta$-keto ester enolates

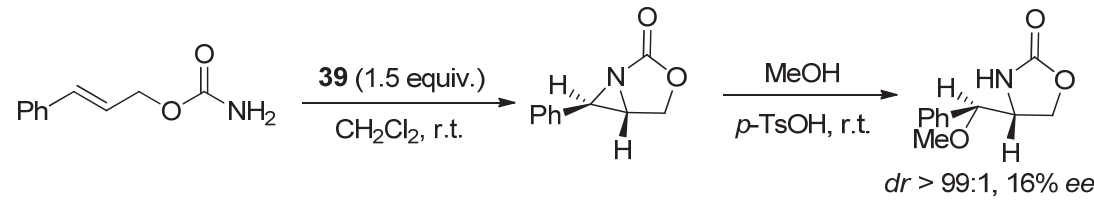

图 31 烯烃的氮杂环丙烷化反应

Figure 31 Aziridination of alkenes 
三价及五价碘试剂, 其中手性五价碘 41 在苯酚类化合 物的不对称去芳构羟基化/[4+2]二聚反应中表现优异, 对映选择性最高可以达到 $94 \%$ ee .

2017 年, Quideau 等 ${ }^{[58}$ 进一步发展了基于联苯骨架 的炔基化试剂 42, 用于 $\beta$-酮酯的 $\alpha$-位不对称炔基化反 应时, 大多情况下收率较高, 对映选择性中等, 最高为 $68 \%$ ee. 他们也尝试利用这类试剂在菜酚底物中实现不 对称炔基化反应, 同样, 大多数情况下收率较好, 但对 映选择性只能达到中等(图 33).

2013 年, Berthiol 和 Einhorn 等 ${ }^{[59]}$ 报道了 3,3'-二碘联䒺酚并马来酰亚胺作为骨架的芳基碘化物, 并在亚胺 上引入带手性取代基的苯环结构, 代表性化合物为结构 43. 他们利用这类催化剂研究羰基化合物的不对称 $\alpha$-磺 酸酯化反应, 但这类复杂的催化剂结构并未给反应带来 高的对映选择性.

2017 年, Dohi和 Kita 等 ${ }^{[00]}$ 报道了使用联萗骨架手性 芳基碘化物 44 和 $m$-CPBA 促进的萗酚化合物的不对称 去芳构化螺环内酯化反应, 当使用当量的芳基碘化物 时, 对映选择性最高可以达到 $76 \% \mathrm{ee}$, 而使用催化量的 芳基碘化物, 对映选择性最高为 $64 \%$ ee. Masson 等 ${ }^{[61]}$ 则 发展了非 $\mathrm{C} 2$ 对称的联苯手性芳基碘化合物 $\mathbf{4 5}$, 催化羰
基的 $\alpha$-磺酸酯化和膦酰酯化反应，对映选择性也在 $60 \% \sim 70 \%$ ee 左右.

总体而言，虽然联菜或联苯骨架的手性配体在过渡 金属催化的反应中, 具有广泛的应用. 但基于联荎或联 苯骨架发展的手性芳基碘试剂或前体，在不对称转化中 的表现差强人意. 这也反映出手性高价碘化学与传统的 过渡金属化学在反应控制等方面具有很大不同，仍然需 要对反应机理等更深入的了解.

\subsection{2 基于手性螺环骨架发展的手性芳基碘试剂或前} 体

由周其林等 ${ }^{\left[{ }^{[2]}\right.}$ 发展的手性螺二氢狮配体在过渡金 属催化的反应中得到了广泛的应用. 螺二氢狮刚性骨架 结构极具特色, 在不对称合成领域引起了极大的研究兴 趣. 基于手性螺二氢茆骨架发展的高价碘在过去十年间 也得以发展, 成为一类重要的手性芳基碘试剂(图 34).

2008 年, Kita 等 ${ }^{[63]}$ 报道了螺二氢荫骨架的手性芳基 碘(III)试剂 46 促进菜酚的首次不对称去芳构螺环内酯 化反应(图 35), 这一反应也被称为 Kita 氧化去芳构螺环 化反应. 产物对映选择性高达 $86 \%$ ee . 而以 $m$-CPBA 为 共氧化剂，以相应的手性芳基碘(I)化合物 $47 \mathbf{a}$ 作为催化
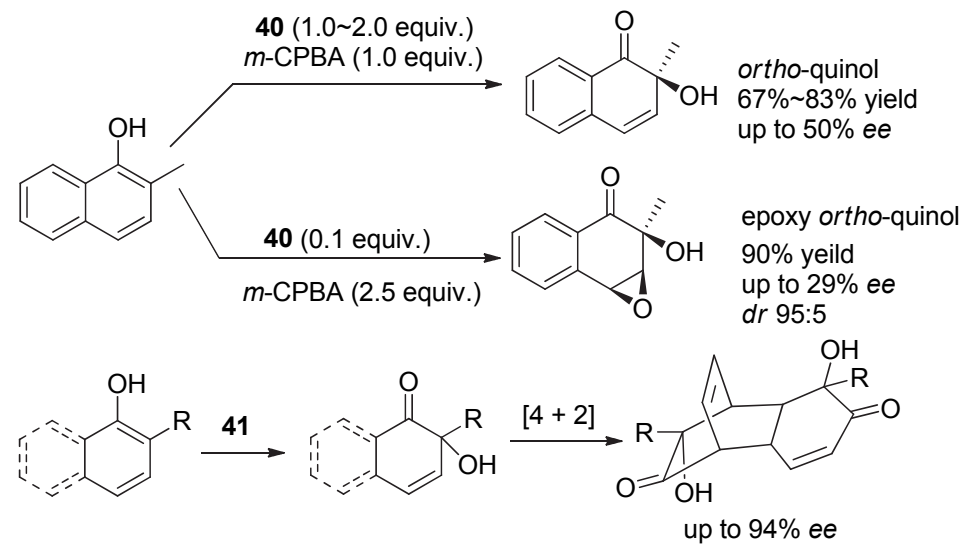

图 32 不对称去芳构化反应研究

Figure 32 Asymmetric dearomatization

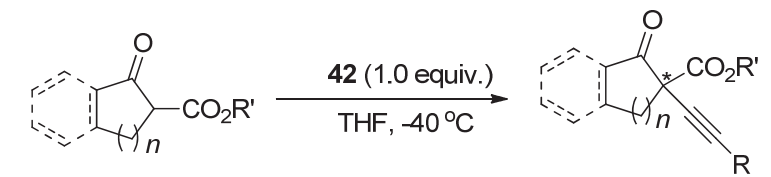

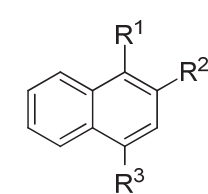

$\alpha$ - or $\beta$-naphthols

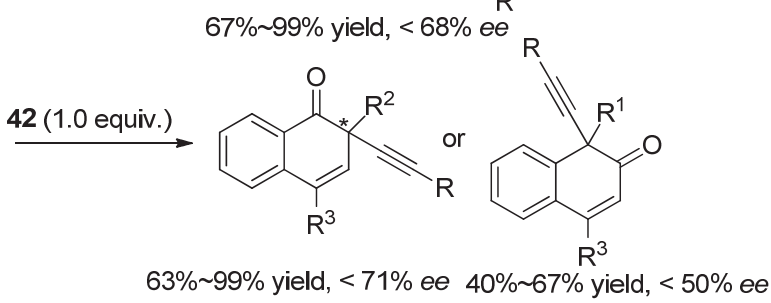

图 33 不对称炔基化反应

Figure 33 Asymmetric alkynylation 


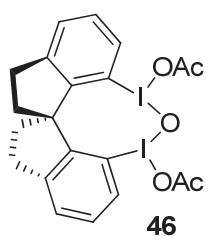

Kita 2008, 2013

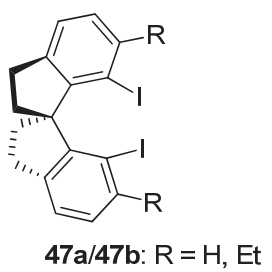

47a/47b: $\mathrm{R}=\mathrm{H}, \mathrm{Et}$

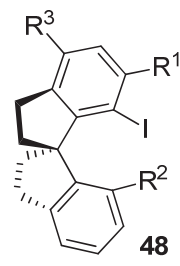

Zhang 2011

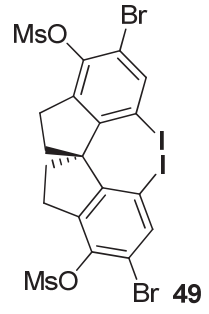

Cai 2018

图 34 基于螺环骨架的手性芳基碘

Figure 34 Spirocyclic skeleton-based chiral aryliodine reagents<smiles>[R]c1cc(CCC(=O)O)c(O)c2ccccc12</smiles>

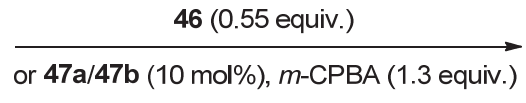<smiles>[R]CC12CCC(CC1)C(=O)O2</smiles>

with 46: $30 \% \sim 87 \%$ yield, up to $86 \%$ ee 47a: $13 \% \sim 75 \%$ yield, up to $69 \%$ ee 47b: $16 \% \sim 90 \%$ yield, up to $92 \%$ ee

图 35 基于螺环结构的手性芳基碘在 Kita 氧化去芳构螺环内酯化反应中的研究

Figure 35 Spirobiindane-based chiral aryliodine reagents and the applications in Kita oxidative spirocyclizations

剂, 催化的同一反应, 对映选择性虽然不如使用碘(III) 试剂, 但同样表现较好, 对映体选择性达到 $69 \%$ ee. 2013 年, 他们 ${ }^{[64]}$ 通过进一步在螺环上引入取代基, 发展 了一系列螺二狮骨架的芳基碘化物. 并以邻位乙基取代 的手性碘化物 $47 \mathrm{~b}$ 为催化剂, 将菜酚的不对称去芳构螺 环内酯化反应 $e e$ 值提高到了 $90 \%$ 以上. 但在大多数情 况下, 反应的收率都只能达到中等. 这一类催化剂可与 后来由 Ishihara 等基于乳酸手性发展的芳基碘化物相媲 美，是两类各有特色的手性芳基碘化合物，在䒺酚的不 对称去芳构螺内酯化反应中取得了重大突破.

这类基于手性螺环的芳基碘化物也被其他研究小 组运用到其它类型的不对称反应研究. 例如, 2011 年, 张弛等 ${ }^{\left[{ }^{65]}\right.}$ 报道了一系列螺二氢茆骨架的手性芳基单碘 化物的合成, 并尝试用于羰基化合物的 $\alpha$-磺酸酯化, 但 对映选择性最高只有 $50 \%$ ee 左右. 2018 年, 蔡倩等 ${ }^{[66]}$ 报道了螺二氢狮骨架的手性芳基碘化物催化的不对称 去对称化 $\mathrm{C}-\mathrm{N}$ 氧化偶联反应，代表性结构为化合物 49. 根据芳环取代基的不同, 可以生成手性四氢喹啉或带中 心手性的螺环内酰胺, 产率中等到良好, 对映选择性最 高分别达到 $89 \% e e$ 和 $81 \% e e$ (图 36).

\subsection{3 基于平面手性环蕃发展的手性芳基碘化物}

最近, 南京大学的郑文华等 ${ }^{[67]}$ 报道了一类基于环 蕃结构的新型平面手性碘代芳烃试剂, 使用[2.2]二聚对 二甲苯为母核, 通过手性柱拆分分离技术, 构建了一系
列手性芳基碘化合物，其代表性结构为化合物 $\mathbf{5 0}$ (图 37). 他们利用这类手性芳基碘化物，应用于 $\beta$-酮酯的亲 核氟化反应，对映选择性最高可达到 $92 \% e e$. 但美中不 足的是, 由于副反应影响, 这一反应收率较低. 但作为 首例平面手性芳基碘催化的高对映选择性反应，这一类 手性芳基碘化物的发展，具有开创性意义.

\subsection{4 其它类型的刚性手性芳基碘化物}

除了上述基于联菜、联苯骨架、螺环骨架、环蕃骨 架发展的刚性手性芳基碘试剂外，文献中还有一些具有 刚性结构的手性芳基碘试剂值得加以注意.

2012 年, Ibrahim 等 ${ }^{[68]}$ 报道了一类桥环八氢葱手性 芳基碘化物, 这类结构中仍为中心手性，但由于骨架为 刚性结构, 我们在本节进行介绍. 其代表性结构为化合 物 51 53(图 38). 这类结构可以促进一系列反应, 如羰 基的 $\alpha$-官能团化等, 但大多对映选择性不高. 在不对称 Kita 氧化去芳构螺环内酯化反应中, 表现一般, 最高仅 获得 $67 \%$ ee.

2017 年, Ogasawara 和 Dohi 等 ${ }^{[69]}$ 报道了一类非常有 意思的阻旋手性有机碘试剂, 代表性结构为 $\mathbf{4 5}$, 这是一 类旋转受阻的二碘代 1,3-二烯类化合物. 这是首例应用 于不对称反应研究的非芳基有机碘化合物, 这类手性烯 基碘化物在 $m$-CPBA 作用下，催化菜酚的 Kita氧化不对 称去芳构螺环化反应，对映选择性可以达到 $73 \% e e$ (图 39).

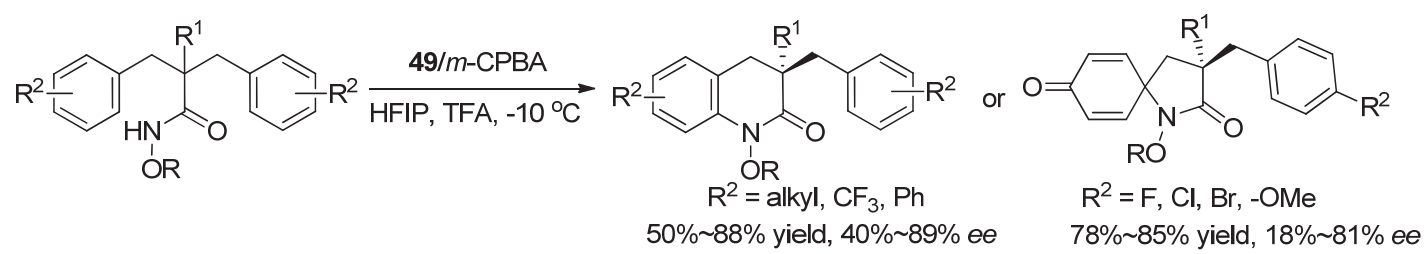

图 36 氧化不对称 $\mathrm{C}-\mathrm{N}$ 偶联反应

Figure 36 Asymmetric oxidative $\mathrm{C}-\mathrm{N}$ coupling 


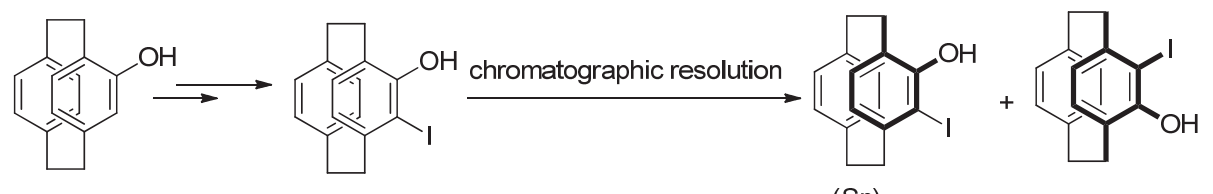

racemic

$(S p)$

$(R p)$

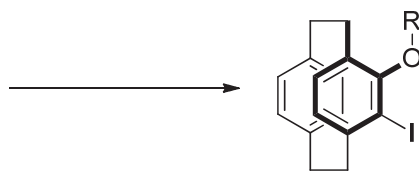

$(S p)$

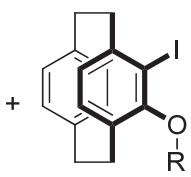

$(R p)$

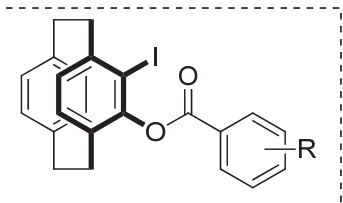

50: $R=3,4,5$-trimethoxy Zheng 2018

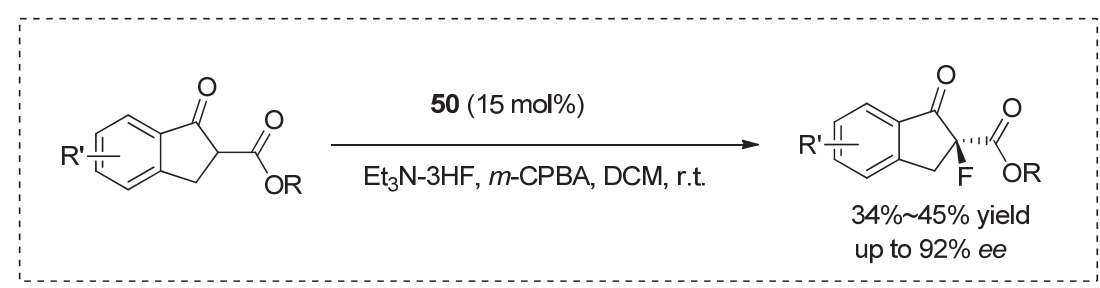

图 37 郑文华组发展的平面手性芳基碘化物

Figure 37 Planar chiral iodoarenes by Zheng et al.

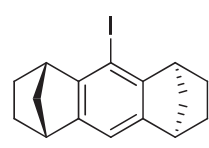

51

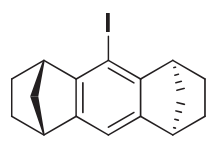

52

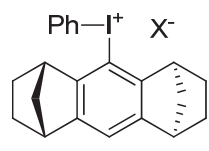

53

图 $38 \mathrm{Ibrahim}$ 组发展的手性高价碘试剂和前体

Figure 38 Chiral hypervalent iodines and precursors by Ibrahim

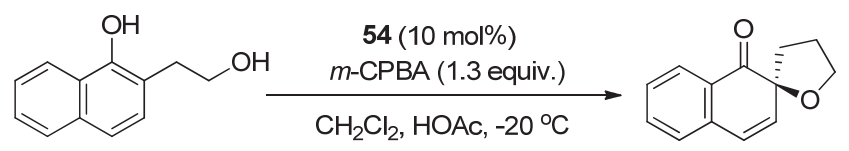

$85 \%$ yield, $73 \%$ ee

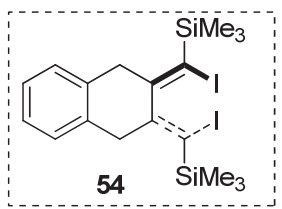

图 39 基于阻旋受阻的手性烯基碘化合物

Figure 39 Atropisomeric chiral diiododienes

\section{3 总结与展望}

经过几十年的发展, 高价碘化学已成为有机合成中 重要的合成工具. 手性有机碘化物在不对称合成中的研 究和应用也日渐广泛. 在这篇综述中, 我们针对不同的 骨架回顾了手性芳基碘试剂的发展以及它们在各类反 应的一些特点. 早期的手性芳基碘试剂是基于配体形式 的高价碘试剂, 由碘原子与手性部位如手性樟脑磺酸、 酒石酸等直接相连. 这类芳基碘试剂主要用于硫醚不对 称氧化成亚砜的反应, 但通常显示较低的对映选择性. 之后在多个研究小组的努力下, 基于手性骨架发展的芳 基碘试剂或前体得以发展和应用. 其中, 基于柔性骨架 中心手性发展的手性高价碘试剂或前体, 取得了巨大的 成功. 特别是 Fujita和 Ishihara等利用乳酸发展的手性高 价碘试剂或前体，在不对称去芳构化、羰基 $\alpha$-位官能团 化、烯烃双官能团化、烯烃氧化重排、氧化偶联等多种
类型的不对称转化中都取得了高的对映选择性. 此外, 基于刚性骨架发展的手性高价碘试剂或前体，也得到了 很好的发展，特别是 Kita 等基于螺二狮骨架的手性高价 碘试剂和前体，以及郑文华等基于环蕃结构发展的平面 手性芳基碘，在不对称去芳构螺环化、氧化偶联、羰基 $\alpha$-位氟化等反应中，展现出优良的对映选择性.

但是，对于手性高价碘促进的不对称转化而言，目 前还处于比较初始的阶段, 具有很大的研究空间. 分析 文献报道的各类手性高价碘试剂及反应，我们认为至少 在以下几个方面值得深入研究:

(1) 对于反应机理以及手性控制的微妙因素的认识 还不够深入, 需要进一步的理论研究和实验验证. 比如, 在反应活性以及对映选择性控制方面，手性高价碘中芳 环上的电性以及碘原子周围的化学环境对反应活性和 对映选择性都具有重要影响, 有必要加以深入研究. 与 过渡金属配体不同，由于碘原子半径较大，邻位位阻太 
大, 可能影响底物与碘结合, 如何选择合适的邻位基团 是需要考虑的重要因素. 此外, 高价碘促进的反应, 往 往存在多种类型的副反应, 反应收率欠佳. 因此, 如何 进行区域选择性控制以及副反应控制等，也存在极大的 研究空间.

(2) 反应类型及底物范围还有待进一步扩展. 高价 碘促进的非手性的反应非常广泛, 而对于不对称转化, 从早期对硫醚的不对称氧化开始, 目前已经有多种类型 的反应可以通过手性高价碘试剂得以实现. 但与过渡金 属催化的不对称反应相比, 反应类型仍然非常局限, 有 很大的拓展空间. 此外, 不少手性高价碘促进的反应, 底物范围受到严重限制, 如何拓展底物适用范围, 也是 一个需要发展的重要方面.

(3) 具有新骨架的手性高价碘试剂发展有待进一步 加强. 尽管目前已有数十种手性碘试剂, 但真正在不对 称转化中能获得高对映选择性的还很少. 新的手性高价 碘试剂, 对于拓展反应类型以及实现高对映选择性转 化, 最为关键. 因此有必要继续设计、发展新骨架的手 性高价碘.

手性高价碘化学作为一个新的研究领域, 已经取得 了巨大的发展, 特别是近十年来, 进展显著. 我们相信, 随着研究的不断深入, 新骨架手性高价碘试剂的发展以 及新不对称转化类型的拓展, 将取得更令人鼓舞的成 果, 在绿色不对称合成中发挥越来越重要的作用.

\section{作者简介}

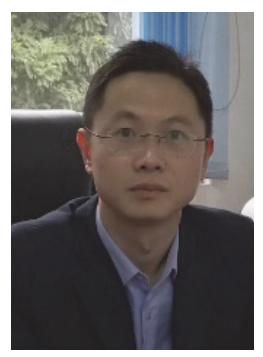

蔡倩, 暨南大学药学院研究员. 2001 年本科毕业于南开大 学, 2006 年从上海有机所获博士学位, 之后在密歇根大学癌症 研究中心从事博士后研究, 2009 年加入中科院广州生物医药 与健康研究院, 2010 年担任课题组长, 2016 年加入暨南大学药 学院. 蔡倩课题组研究兴趣集中在基于药物活性分子合成的 新型串联反应以及不对称反应方法学研究及应用.

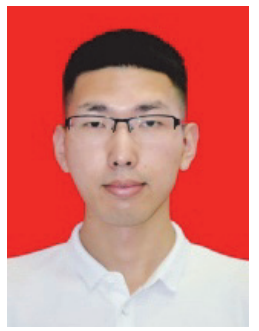

马浩文, 1996 年生于湖北省黄石市, 2018 年本科毕业于山 西医科大学药学院, 现为暨南大学药学院药物化学专业硕士 研究生, 主要研究课题为高价碘促进的不对称反应.

\section{References}

[1] For books, see: (a) Chemistry of Hypervalent Compounds, Ed.: AKiba, K. Y., Wiley-VCH, New York, 1999. (b) Zhdankin, V. V. Hypervalent Iodine Chemistry: Preparation, Structure and Synthetic Application of Polyvalent Iodine Compounds, John Wiley \& Sons Ltd., New York, 2014. (c) Iodine Chemistry And Applications, Ed. Kaiho, T., John Wiley \& Sons Ltd., New York, 2015. (d) Hypervalent Iodine Chemistry: Modern Developments in Organic Synthesis, Ed.: Wirth, T., Springer, 2003.

[2] For recent reviews, see: (a) Yoshimura, A.; Zhdankin, V. V. Chem. Rev. 2016, 116, 3328. (b) Duan, Y.; Jiang, S.; Han, Y.; Sun, B.; Zhang, C. Chin. J. Org. Chem. 2016, 36, 1973 (in Chinese). (段亚 南, 姜山, 韩永超, 孙博, 张弛, 有机化学, 2016, 36, 1973.) (c) Ma, J.; Chen, L.; Yuan, Z.; Cheng, H. Chin. J. Org. Chem. 2018, 38, 1586 (in Chinese). (马姣丽, 陈立成, 袁中文, 程辉成, 有机化学, 2018, 38, 1586.)

[3] For selected recent reviews, see: (a) Flores, A.; Cots, E.; Bergès, J.; Muñiz, K. Adv. Synth. Catal. 2019, 361, DOI:10.1002/adsc. 201800521. (b) Martín Romero, R.; Wöste, T. H.; Muñiz, K. Chem. Asian J. 2014, 9, 972. (c) Singh, F. V.; Wirth, T. Chem. Asian J. 2014, 9, 950. (d) Harned, A. M. Tetrahedron Lett. 2014, 55, 4681. (e) Parra, A.; Reboredo, S. Chem. Eur. J. 2013, 19, 17244.

[4] Liang, H.; Ciufolini, M. A. Angew. Chem. Int. Ed. 2011, 50, 11849.

[5] Ochiai, M.; Takeuchi, Y.; Katayama, T.; Sueda, T.; Miyamoto, K. J. Am. Chem. Soc. 2005, 127, 12244. (b) Dohi, T.; Maruyama, A.; Yoshimura, M.; Morimoto, K.; Tohma, H.; Kita, Y. Angew. Chem. Int. Ed. 2005, 44, 6193.

[6] Pribram, R. Justus Liebigs Ann. Chem. 1907, 351, 481.

[7] Imamoto, T.; Koto, H. Chem. Lett. 1986, 967.

[8] Hatzigrigoriou, E.; Varvoglis, A.; Bakola-Christianopoulou, M. J. Org. Chem. 1990, 55, 315.

[9] Xia, M.; Chen, Z.-C. Synth. Commun. 1997, 27, 1321.

[10] Ray III, D. G.; Koser, G. F. J. Am. Chem. Soc. 1990, 112, 5672.

[11] Ray III D. G.; Koser, G. F. J. Org. Chem. 1992, 57, 1607.

[12] Tohma, H.; Takizawa, S.; Watanabe, H.; Fukuoka, Y.; Maegawa, T.; Kita, Y. J. Org. Chem. 1999, 64, 3519.

[13] Rabah, G. A.; Koser, G. F. Tetrahedron Lett. 1996, 37, 6453.

[14] (a) Wirth, T.; Hirt, U. H. Tetrahedron Asymmetry 1997, 8, 23. (b) Hirt, U. H.; Spingler, B.; Wirth, T. J. Org. Chem. 1998, 63, 7674. (c) Hirt, U. H.; Schuster, M. F. H.; French, A. N.; Wiest, O. G.; Wirth, T. Eur. J. Org. Chem. 2001, 1569.

[15] Mizar, P.; Laverny, A.; EI-Sherbini, M.; Farid, U.; Brown, M.; Malmedy, F.; Wirth, T. Chem. Eur. J. 2014, 20, 9910.

[16] Hempel, C.; Maichle-Mössmer, C.; Pericàs, M. A.; Nachtsheim, B. J. Adv. Synth. Catal. 2017, 359, 2941.

[17] Fujita, M.; Okuno, S.; Lee, H. J.; Sugimura, T.; Okuyama, T. Tetrahedron Lett. 2007, 48, 8691.

[18] (a) Uyanik, M.; Yasui, T.; Ishihara, K. Angew. Chem. Int. Ed. 2010, 49, 2175. (b) Uyanik, M.; Yasui, T.; Ishihara, K. Tetrahedron 2010, 66,5841 .

[19] (a) Fujita, M.; Yoshida, Y.; Miyata, K.; Wakisaka, A.; Sugimura, T. Angew. Chem. Int. Ed. 2010, 49, 7068. (b) Fujita, M.; Mori, K.; Shimogaki, M.; Sugimura, T. Org. Lett. 2012, 14, 1294. (c) Shimogaki, M.; Fujita, M.; Sugimura, T. Eur. J. Org. Chem. 2013, 7128. (d) Takesue, T.; Fujita, M.; Sugimura, T.; Akutsu, H. Org. Lett. 2014, 16,4634

[20] Fujita, M.; Wakita, M.; Sugimura, T. Chem. Commun. 2011, 47, 3983.

[21] (a) Shimogaki, M.; Fujita, M.; Sugimura, T. Angew. Chem. Int. Ed. 2016, 55, 15797. (b) Shimogaki, M.; Fujita, M.; Sugimura, T. J. Org. Chem. 2017, 82, 11836.

[22] Röben, C.; Souto, J. A.; González, Y.; Lishchynskyi, A.; Muñiz, K. Angew. Chem. Int. Ed. 2011, 50, 9478.

[23] Muñiz, K.; Barreiro, L.; Romero, R. M.; Martínez, C. J. Am. Chem. Soc. 2017, 139, 4354.

[24] (a) Haubenreisser, S.; Wöste, T. H.; Martínez, C.; Ishihara, K.; Muñiz, K. Angew. Chem. Int. Ed. 2016, 55, 413. (b) Wöste, T. H.; Muñiz, K. Synthesis 2016, 48, 816.

[25] (a) Farid, U.; Wirth, T. Angew. Chem. Int. Ed. 2012, 51, 3462. (b) Mizar, P.; Niebuhr, R.; Hutchings, M.; Farooq, U.; Wirth, T. Chem. Eur. J. 2016, 22, 1614.

[26] Gelis, C.; Dumoulin, A.; Bekkaye, M.; Neuville, L.; Masson, G. Org Lett. 2017, 19, 278.

[27] (a) Kong, W.; Feige, P.; de Haro, T.; Nevado, C. Angew. Chem. Int. 
Ed. 2013, 52, 2469. (b) Pluta, R.; Krach, P. E.; Cavallo, L.; Falivene, L.; Rueping, M. ACS Catal. 2018, 8, 2582.

[28] Wu, H.; He, Y.-P.; Xu, L.; Zhang, D.-Y.; Gong, L.-Z. Angew. Chem. Int. Ed. 2014, 53, 3466

[29] Zhang, D.-Y.; Xu, L.; Wu, H.; Gong, L.-Z. Chem. Eur. J. 2015, 21, 10314.

[30] Cao, Y.; Zhang, X.; Lin, G.; Zhang-Negrerie, D.; Du, Y. Org. Lett. 2016, 18,5580 .

[31] Farid, U.; Malmedy, F.; Claveau, R.; Albers, C.; Wirth, T. Angew. Chem. Int. Ed. 2013, 52, 7018 .

[32] Brown, M.; Kumar, R.; Rehbein, J.; Wirth, T. Chem. Eur. J. 2016, 22, 4030

[33] Banik, S. M.; Medley, J. W.; Jacobsen, E. N. J. Am. Chem. Soc. 2016, 138, 5000 .

[34] Banik, S. M.; Medley, J. W.; Jacobsen, E. N. Science 2016, 353, 51.

[35] Zhou, B.; Haj, M. K.; Jacobsen, E. N.; Houk, K. N.; Xue, X.-S. J. Am. Chem. Soc. 2018, 140, 15206.

[36] Mennie, K. M.; Banik, S. M.; Reichert, E. C.; Jacobsen, E. N. J. Am. Chem. Soc. 2018, 140, 4797.

[37] Qurban, J.; Elsherbini, M.; Wirth, T. J. Org. Chem. 2017, 82, 11872.

[38] Hashimoto, T.; Shimazaki, Y.; Omatsu, Y.; Maruoka, K. Angew. Chem. Int. Ed. 2018, 57, 7200.

[39] Zhdandin, V. V.; Smart, J. T.; Zhao, P.; Kiprof, P. Tetrahedron Lett. 2000, 41, 5299.

[40] Ladziata, U.; Carlson, J.; Zhdankin, V. V. Tetrahedron Lett. 2006, 47, 6301.

[41] Altermann, S. M.; Richardson, R. D.; Page, T. K.; Schmidt, R. K.; Holland, E.; Mohammed, U.; Paradine, S. M.; French, A. N.; Richter, C.; Bahar, A. M.; Witulski, B.; Wirth, T. Eur. J. Org. Chem. 2008, 5315.

[42] Farooq, U.; Schäfer, S.; Ali Shah, A.-U.-H.; Freudendahl, D. M.; Wirth, T. Synthesis 2010, 1023.

[43] Volp, K. A.; Harned, A. M. Chem. Commun. 2013, 49, 3001.

[44] Boppisetti, J. K.; Birman, V. B. Org. Lett. 2009, 6, 1221.

[45] Guilbault, A.-A.; Basdevant, B.; Wanie, V.; Legault, C. Y. J. Org. Chem. 2012, 77, 11283.

[46] Rodríguez, A.; Moran, W. J. Synthesis 2012, 44, 1178.

[47] Uyanik, M.; Yasui, T.; Ishihara, K. Angew. Chem. Int. Ed. 2013, 52, 9215.

[48] Uyanik, M.; Sasakura, N.; Mizuno, M.; Ishihara, K. ACS Catal. 2017, 7, 872 .

[49] Uyanik, M.; Yasui, Y.; Ishihara, K. J. Org. Chem. 2017, 82, 11946.

[50] Jain, N.; Xu, S.; Ciufolini, M. A. Chem. Eur. J. 2017, 23, 4542.

[51] Molnár, I. G.; Gilmour, R. J. Am. Chem. Soc. 2016, 138, 5004.
[52] Scheidt, F.; Schäfer, M.; Sarie, J. C.; Doniliuc, C. G.; Molloy, J. J.; Gilmour, R. Angew. Chem. Int. Ed. 2018, 57, 16431.

[53] Ochiai, M.; Takaoka, Y.; Masaki, Y. J. Am. Chem. Soc. 1990, 112, 5677.

[54] Ochiai, M.; Kitagawa, Y.; Takayama, N.; Takaoka, Y.; Shiro, M. J. Am. Chem. Soc. 1999, 121, 9234.

[55] Deng, Q.-H.; Wang, J.-C.; Xu, Z.-J.; Zhou, C.-Y.; Che, C.-M. Synthesis 2011, 18, 2959.

[56] Quideau, S.; Lyvinec, G.; Marguerit, M.; Bathany, K.; Ozanne-Beaudenon, A.; Buffeteau, T.; Cavagnat, D.; Chénedé, A. Angew. Chem. Int. Ed. 2009, 48, 4605.

[57] Bosset, C.; Coffinier, R.; Peixoto, P. A.; Assal, M. E.; Miqueu, K. M.; Sotiropoulos, J.-M. Pouységu, L.; Quideau, S. Angew. Chem. Int. Ed. 2014, 53, 9860 .

[58] Companys, S.; Peixoto, P. A.; Bosset, C.; Chassaing, S.; Miqueu, K.; Sotiropoulos, J.-M.; Pouységu, L.; Quideau, S. Chem. Eur. J. 2017, 23, 13309.

[59] (a) Brenet, S.; Berthiol, F.; Einhorn, J. Eur. J. Org. Chem. 2013, 8094. (b) Brenet S.; Minozzi, C.; Clarens, B.; Amiri, L.; Berthiol, F. Synthesis 2015, 47, 3859

[60] Dohi, T.; Sasa, H.; Miyazaki, K.; Fujitake, M.; Takenaga, N.; Kita, Y. J. Org. Chem. 2017, 82, 11954.

[61] Levitre, G.; Dumoulin, A.; Retailleau, P.; Panossian, A.; Leroux, F. R.; Masson, G. J. Org. Chem. 2017, 82, 11877.

[62] Xue, J.-H.; Zhou, Q.-L. Acta Chim. Sinica 2014, 72, 778 (in Chinese). (谢建华, 周其林, 化学学报, 2014, 72, 778.)

[63] Dohi, T.; Maruyama, A.; Takenaga, N.; Senami, K.; Minamitsuji, Y.; Fujioka, H.; Caemmerer, S. B.; Kita, Y. Angew. Chem. Int. Ed. 2008 47, 3787.

[64] Dohi, T.; Takenaga, N.; Nakae, T.; Toyoda, Y.; Yamasaki, M.; Shiro, M.; Fujioka, H.; Maruyama, A.; Kita, Y. J. Am. Chem. Soc. 2013, $135,4558$.

[65] Yu, J.; Cui, J.; Hou, X.-S.; Liu, S.-S.; Gao, W.-C.; Jiang, S.; Tian, J.; Zhang, C. Tetrahedron: Asymmetry 2011, 22, 2039.

[66] Ding, Q.; He, H.; Cai, Q. Org. Lett. 2018, 20, 4554.

[67] Wang, Y.; Yuan, H.; Lu, H.; Zheng, W.-H. Org. Lett. 2018, 20, 2555.

[68] Murray, S. J.; Müller-Bunz, H.; Ibrahim, H. Chem. Commun. 2012, 48,6268 .

[69] Ogasawara, M.; Sasa, H.; Hu, H.; Amano, Y.; Nakajima, H.; Takenaga, N.; Nakajima, K.; Kita, Y.; Takahashi, T.; Dohi, T. Org. Lett. 2017, 19, 4102 . 